\title{
Supersymmetric Dirac-Born-Infeld axionic inflation and non-Gaussianity
}

\author{
Pran Nath and Maksim Piskunov \\ Department of Physics, Northeastern University, \\ 100 Forsyth St, Boston, MA 02115-5000, U.S.A. \\ E-mail: p.nath@northeastern.edu, m.piskunov@northeastern.edu
}

\begin{abstract}
An analysis is given of inflation based on a supersymmetric Dirac-Born-Infeld (DBI) action in an axionic landscape. The DBI model we discuss involves a landscape of chiral superfields with one $\mathrm{U}(1)$ shift symmetry which is broken by instanton type nonperturbative terms in the superpotential. Breaking of the shift symmetry leads to one pseudo-Nambu-Goldstone-boson which acts as the inflaton while the remaining normalized phases of the chiral fields generically labeled axions are invariant under the $U(1)$ shift symmetry. The analysis is carried out in the vacuum with stabilized saxions, which are the magnitudes of the chiral fields. Regions of the parameter space where slow-roll inflation occurs are exhibited and the spectral indices as well as the ratio of the tensor to the scalar power spectrum are computed. An interesting aspect of supersymmetric DBI models analyzed is that in most of the parameter space tensor to scalar ratio and scalar spectral index are consistent with Planck data if slow roll occurs and is not eternal. Also interesting is that the ratio of the tensor to the scalar power spectrum can be large and can lie close to the experimental upper limit and thus testable in improved experiment. Non-Gaussianity in this class of models is explored.
\end{abstract}

Keywords: Supersymmetry Phenomenology

ARXIV EPRINT: 1807.02549 


\section{Contents}

1 Introduction 1

2 Fast-roll and slow-roll decomposition $\quad 2$

3 Supersymmetric DBI action for two chiral fields 3

4 Pressure, density and inflation equations $\quad 9$

$\begin{array}{lll}5 & \text { Slow roll parameters and non-Gaussianity } & 10\end{array}$

$6 \quad$ Model simulation and experimental test 12

$\begin{array}{lll}7 & \text { Conclusion } & 16\end{array}$

$\begin{array}{ll}\text { A Single field supersymmetric DBI Lagrangian } & 17\end{array}$

$\begin{array}{ll}\text { B Equations for } F_{k}, F_{k}^{*}(k=1,2) & 18\end{array}$

$\begin{array}{ll}\text { C DBI scalar potential with derivative terms absent } & 20\end{array}$

\section{Introduction}

As is well known many of the problems associated with Big Bang cosmology which include the flatness problem, the horizon problem, and the monopole problem are resolved by inflation [1-6]. Quantum fluctuations at the time of horizon exit carry significant information regarding specifics of the inflationary model [7-12] which can be extracted from cosmic microwave background (CMB) radiation anisotropy. The data from the Planck experiment [13-15] has helped constrain inflation models excluding some and narrowing down the parameter space of others. One such model is so called natural inflation based on a U(1) shift symmetry which is described by a simple potential $[16,17] V(a)=\Lambda^{4}\left(1+\cos \left(\frac{a}{f}\right)\right)$, where $a$ is the axion field and $f$ is the axion decay constant. In this case consistency with Planck data requires the axion decay constant to be significantly greater than the Planck mass $M_{\mathrm{P}}$. However, an axion decay constant larger than the Planck mass is undesirable since a global symmetry is not preserved by quantum gravity unless it has a gauge origin. Additionally string theory prefers the axion decay constant to lie below $M_{\mathrm{P}}[18,19]$. It turns out that the reduction of the decay constant poses a problem, and several suggestions exist regarding its resolution such as the so called alignment mechanism [20, 21].

A procedure for resolving the axion decay constant problem was proposed in [22]. One element of this analysis relies on a decomposition of the potential into a fast-roll 
and a slow-roll parts where the slow roll is controlled only by the inflaton field while the remaining fields enter in fast roll and are not relevant for inflation [22] (for a review of inflation in supersymmetric theories see, e.g., [23]). An analysis within this model shows that one can obtain spectral indices as well as the ratio of the tensor to the scalar power spectrum consistent with the Planck data [13-15]. Another quantity of interest in primordial perturbations is the so-called non-Gaussianity [24-29]. It is known that models with canonical kinetic energy do not lead to non-Gaussianity and for non-Gaussianity one needs models with non-canonical kinetic energy. In this context the Dirac-Born-Infeld (DBI) models are of interest (see, e.g., [30-34]) which is the object of study in this work. Our work is focused on using shift symmetry and axions for inflation. For a partial list of other works where shift symmetry of axions is utilized in inflation see [35-41] and in the string context see [42-44]. For reviews of axionic cosmology see [45, 46].

The outline of the rest of the paper is as follows: in section 2 we give a summary of previous results on the decomposition of a landscape of axion fields which undergo shifts under a $\mathrm{U}(1)$ global transformation into fast-roll and slow-roll parts. This is one of the central elements in the analysis of the inflationary models we discuss later. In section 3 we give a description of the supersymmetric DBI Lagrangian in superspace for the case of two chiral fields $\Phi_{1}$ and $\Phi_{2}$ which are oppositely charged under a U(1) global symmetry. We then display the bosonic part of the Lagrangian after integration over the Grassmann coordinates. Here it is shown that the Lagrangian depends on the dimensionless parameters $\alpha_{1}, \alpha_{2}, \alpha_{3}$; and $T$ which has the dimension of the fourth power of mass. The general case including $\alpha_{1}, \alpha_{2}, \alpha_{3}$ is too complicated to discuss analytically and thus here the analysis is given taking into account only the $\alpha_{1}$ terms. In section 4 we discuss the pressure, density and the inflation equations for a generic DBI model. In section 5 we discuss the slow-roll parameters, non-Gaussianity, and the speed of sound which enters in defining non-Gaussianity. Model simulations and experimental test of the two field DBI model is discussed in section 6 , and conclusions are given in section 7 . Further, details of the analysis are given in appendices A, B, and C.

\section{Fast-roll and slow-roll decomposition}

Before discussing the supersymmetric DBI model we summarize first the slow-roll and fast-roll decomposition of the inflation potential which is one of the central components of the analysis of this paper for the DBI case. As noted above the slow-roll and fast-roll decomposition of the potential was introduced in [22]. This analysis utilizes a landscape of pairs of chiral fields which are charged under a U(1) global symmetry. Thus suppose we have a set of chiral fields $\Phi_{i}(i=1, \cdots, n)$ where $\Phi_{i}$ carry the same charge under the shift symmetry and the fields $\tilde{\Phi}_{i}(i=1, \cdots, n)$ carry the opposite charge. We assume that under $\mathrm{U}(1)$ transformations the fields transform as follows

$$
\Phi_{i} \rightarrow e^{i q \lambda} \Phi_{i}, \quad \tilde{\Phi}_{i} \rightarrow e^{-i q \lambda} \tilde{\Phi}_{i}, \quad i=1, \cdots, n .
$$

The superfields $\Phi_{i}$ have an expansion,

$$
\Phi_{i}=\phi_{i}+\theta \chi_{i}+\theta \theta F_{i},
$$


where $\phi_{i}$ is a complex scalar field consisting of the saxion (the magnitude) and the axion (the normalized phase), $\chi_{i}$ is the axino, and $F_{i}$ is an auxiliary field. Similarly the superfields $\tilde{\Phi}_{i}$ have an expansion: $\tilde{\Phi}_{i}=\tilde{\phi}_{i}+\tilde{\theta} \tilde{\chi}_{i}+\tilde{\theta} \tilde{\theta} \tilde{F}_{i}$. We may parametrize $\phi_{i}$ and $\tilde{\phi}_{i}$ so that

$$
\phi_{i}=\frac{1}{\sqrt{2}}\left(f_{i}+\rho_{i}\right) e^{i a_{i} / f_{i}}, \quad \tilde{\phi}_{i}=\frac{1}{\sqrt{2}}\left(\tilde{f}_{i}+\tilde{\rho}_{i}\right) e^{i \tilde{a}_{i} / \tilde{f}_{i}},
$$

where $f_{i}=\left\langle\phi_{i}\right\rangle, \tilde{f}_{i}=\left\langle\tilde{\phi}_{i}\right\rangle$ and $\left(\rho_{i}, a_{i}\right)$ and $\left(\tilde{\rho}_{i}, \tilde{a}_{i}\right)$ are the fluctuations of the quantum fields around their vacuum expectation values $f_{i}, \tilde{f}_{i}$. The above constitute $2 n$ number of axionic fields $a_{1}, \cdots, a_{n}$ and $\tilde{a}_{1}, \cdots, \tilde{a}_{n}$. Since there is only one $\mathrm{U}(1)$ shift symmetry, we can pick a basis where only one linear combination of it is variant under the shift symmetry and all others are invariant. We label this new basis $a_{-}, a_{+}, b_{1}, b_{2}, \cdots, b_{n-1}, \tilde{b}_{1}, \tilde{b}_{2}, \cdots, \tilde{b}_{n-1}$ where only $a_{-}$is sensitive to the shift symmetry. Thus the object of central interest is the field $a_{-}$which is the pseudo-Nambu-Goldstone-Boson (pNBG) and acts as the inflaton. It can be expressed in terms of the original set of axion fields as below

$$
\begin{aligned}
a_{-} & =\frac{1}{f_{e}}\left(\sum_{i=1}^{m} f_{i} a_{i}-\sum_{i=1}^{m} \tilde{f}_{i} \tilde{a}_{i}\right), \\
f_{e} & =\sqrt{\sum_{i=1}^{m} f_{i}^{2}+\sum_{i=1}^{m} \tilde{f}_{i}^{2}} .
\end{aligned}
$$

The relation eq. (2.5) was derived in [22] (see also [47]). The result of eq. (2.5) gives $f_{e}=\sqrt{N} f$ for the case when $f_{i}=\tilde{f}_{i}=f$ and $N=2 m$ which is the N-flation result but derived here in a different context [48, 49].

\section{Supersymmetric DBI action for two chiral fields}

Supersymmetric DBI actions have been investigated by a number of authors (see, e.g., [50$59])$. Here we discuss the supersymmetric DBI in the context of axion inflation. The case of a single field DBI is given in appendix A. Here we consider a pair of chiral superfields $\Phi_{1}$ and $\Phi_{2}$ which carry opposite charges under a global U(1) symmetry. The supersymmetric Lagrangian involving $\Phi_{1}$ and $\Phi_{2}$ is given by

$$
\mathcal{L}=\mathcal{L}_{D}+\mathcal{L}_{F}
$$

where $\mathcal{L}_{D}$ is the D-part of the Lagrangian and $\mathcal{L}_{F}$ is the F-part. We consider the D-part consisting of a part $\mathcal{L}_{D}^{(1)}$ which is quadratic in the fields and a part $\mathcal{L}_{D}^{(2)}$ which is quartic in the fields so that

$$
\mathcal{L}_{D}=\mathcal{L}_{D}^{(1)}+\mathcal{L}_{D}^{(2)}
$$

where $\mathcal{L}_{D}^{(1)}$ and $\mathcal{L}_{D}^{(2)}$ are invariant under the $\mathrm{U}(1)$ symmetry and are given by

$$
\mathcal{L}_{D}^{(1)}=\int d^{4} \theta\left(\Phi_{1} \Phi_{1}^{\dagger}+\Phi_{2} \Phi_{2}^{\dagger}\right)
$$


and

$$
\mathcal{L}_{D}^{(2)}=\mathcal{L}_{D}^{(2 a)}+\mathcal{L}_{D}^{(2 b)}+\mathcal{L}_{D}^{(2 c)}+\mathcal{L}_{D}^{(2 d)}+\mathcal{L}_{D}^{(2 e)}
$$

where

$$
\begin{aligned}
\mathcal{L}_{D}^{(2 a)} & =\int d^{4} \theta \frac{\alpha_{1}}{16 T}\left(D^{\alpha} \Phi_{1} D_{\alpha} \Phi_{1}\right)\left(\bar{D}^{\dot{\alpha}} \Phi_{1}^{\dagger} \bar{D}_{\dot{\alpha}} \Phi_{1}^{\dagger}\right) G(\phi), \\
\mathcal{L}_{D}^{(2 b)} & =\int d^{4} \theta \frac{\alpha_{1}}{16 T}\left(D^{\alpha} \Phi_{2} D_{\alpha} \Phi_{2}\right)\left(\bar{D}^{\dot{\alpha}} \Phi_{2}^{\dagger} \bar{D}_{\dot{\alpha}} \Phi_{2}^{\dagger}\right) G(\phi), \\
\mathcal{L}_{D}^{(2 c)} & =\int d^{4} \theta \frac{\alpha_{2}}{16 T}\left(D^{\alpha} \Phi_{1} D_{\alpha} \Phi_{1}\right)\left(\bar{D}^{\dot{\alpha}} \Phi_{2}^{\dagger} \bar{D}_{\dot{\alpha}} \Phi_{2}^{\dagger}\right) G(\phi), \\
\mathcal{L}_{D}^{(2 d)} & =\int d^{4} \theta \frac{\alpha_{2}}{16 T}\left(D^{\alpha} \Phi_{2} D_{\alpha} \Phi_{2}\right)\left(\bar{D}^{\dot{\alpha}} \Phi_{1}^{\dagger} \bar{D}_{\dot{\alpha}} \Phi_{1}^{\dagger}\right) G(\phi), \\
\mathcal{L}_{D}^{(2 d)} & =\int d^{4} \theta \frac{\alpha_{3}}{16 T}\left(D^{\alpha} \Phi_{1} D_{\alpha} \Phi_{2}\right)\left(\bar{D}^{\dot{\alpha}} \Phi_{1}^{\dagger} \bar{D}_{\dot{\alpha}} \Phi_{2}^{\dagger}\right) G(\phi) .
\end{aligned}
$$

Here

$$
G(\phi)=\frac{1}{T} \frac{1}{1+A+\sqrt{(1+A)^{2}-B}},
$$

and $A$ and $B$ are assumed to have the following forms

$$
\begin{aligned}
A= & \left(\partial_{a} \phi_{1} \partial^{a} \phi_{1}^{*}+\partial_{a} \phi_{2} \partial^{a} \phi_{2}^{*}\right) / T, \\
B= & \left(\alpha_{1} \partial_{a} \phi_{1} \partial^{a} \phi_{1} \partial_{b} \phi_{1}^{*} \partial^{b} \phi_{1}^{*}+\alpha_{1} \partial_{a} \phi_{2} \partial^{a} \phi_{2} \partial_{b} \phi_{2}^{*} \partial^{b} \phi_{2}^{*}+\alpha_{2} \partial_{a} \phi_{1} \partial^{a} \phi_{1} \partial_{b} \phi_{2}^{*} \partial^{b} \phi_{2}^{*}\right. \\
& \left.+\alpha_{2} \partial_{a} \phi_{2} \partial^{a} \phi_{2} \partial_{b} \phi_{1}^{*} \partial^{b} \phi_{1}^{*}+\alpha_{3} \partial_{a} \phi_{1} \partial^{a} \phi_{2} \partial_{b} \phi_{1}^{*} \partial^{b} \phi_{2}^{*}\right) / T^{2},
\end{aligned}
$$

where the supercovariant derivatives $D^{\alpha} \Phi$ etc are defined in an explicit manner in appendix A. We note that the Lagrangian of eq. (3.5) is a direct generalization of the Lagrangian for the single field case (see appendix A) which can be derived from a more basic 3-brane action (see, e.g., [54, 55, 58] and the references therein). Here we simply extend the analysis to two fields in the most general supersymmetric form involving four covariant derivatives. In writing eq. (3.5) we imposed an additional constraint which is invariance under $\Phi_{1}$ and $\Phi_{2}$ interchange. The possible relation of this Lagrangian to an underlying string model is an open question. Here we simply treat eq. (3.5) as an effective low energy theory. Finally $\mathcal{L}_{F}$ is given by

$$
\mathcal{L}_{F}=\int d^{2} \theta W\left(\Phi_{1}, \Phi_{2}\right)+\int d^{2} \bar{\theta} W^{*}\left(\Phi_{1}^{\dagger}, \Phi_{2}^{\dagger}\right)
$$

where the superpotential $W$ is given by

$$
W=W_{s}+W_{s b} .
$$

Here $W_{s}$ is invariant under the global $\mathrm{U}(1)$ symmetry and is taken to be of the form

$$
W_{s}=\mu \Phi_{1} \Phi_{2}+\frac{\lambda}{2}\left(\Phi_{1} \Phi_{2}\right)^{2}
$$


The form eq. (3.10) is chosen so that we can stabilize the saxion VEVs. Eq. (3.10) also explains why we need a pair of chiral fields with opposite $\mathrm{U}(1)$ charges because with a single chiral field which is charged under a U(1) symmetry we cannot form a non-trivial $W_{s}$, needed for stabilizing the saxions, which is invariant under the $\mathrm{U}(1)$ symmetry. $W_{s b}$ breaks the global U(1) symmetry and is taken to be of the form

$$
W_{s b}=\sum_{k=1}^{m}\left(A_{1, k} \Phi_{1}^{k}+A_{2, k} \Phi_{2}^{k}\right) .
$$

We note in passing that the superpotential of the type eqs. (3.9)-(3.11) was considered in $[22,60]$. Integration over $\theta^{\prime} s$ gives for the full Lagrangian

$$
\begin{aligned}
\mathcal{L}= & \mathcal{L}_{D}+\mathcal{L}_{F}=\mathcal{L}_{I}+\mathcal{L}_{I I}, \\
\mathcal{L}_{I}= & T-T \sqrt{(1+A)^{2}-B}, \\
\mathcal{L}_{I I}= & F_{1} F_{1}^{*}+F_{2} F_{2}^{*}+G(\phi)\left[\alpha_{1}\left(-2 F_{1} F_{1}^{*} \partial_{a} \phi_{1} \partial^{a} \phi_{1}^{*}+F_{1}^{2} F_{1}^{* 2}\right)+\alpha_{1}\left(-2 F_{2} F_{2}^{*} \partial_{a} \phi_{2} \partial^{a} \phi_{2}^{*}\right.\right. \\
& \left.+F_{2}^{2} F_{2}^{* 2}\right)+\alpha_{2}\left(-2 F_{1} F_{2}^{*} \partial_{a} \phi_{1} \partial^{a} \phi_{2}^{*}+F_{1}^{2} F_{2}^{* 2}-2 F_{2} F_{1}^{*} \partial_{a} \phi_{2} \partial^{a} \phi_{1}^{*}+F_{2}^{2} F_{1}^{* 2}\right) \\
& \left.+\alpha_{3}\left(-F_{1} F_{1}^{*} \partial_{a} \phi_{2} \partial^{a} \phi_{2}^{*}-F_{2} F_{2}^{*} \partial_{a} \phi_{1} \partial^{a} \phi_{1}^{*}+F_{1} F_{2} F_{1}^{*} F_{2}^{*}\right)\right]+\left(\frac{\partial W}{\partial \phi_{1}} F_{1}+\frac{\partial W}{\partial \phi_{2}} F_{2}+\text { h.c. }\right),
\end{aligned}
$$

where $\frac{\partial W}{\partial \phi_{k}}(k=1,2)$ are given by

$$
\begin{aligned}
& \frac{\partial W}{\partial \phi_{1}}=\mu \phi_{2}+\lambda\left(\phi_{1} \phi_{2}\right) \phi_{2}+\sum_{k=1}^{m} k A_{1, k} \phi_{1}^{k-1}, \\
& \frac{\partial W}{\partial \phi_{2}}=\mu \phi_{1}+\lambda\left(\phi_{1} \phi_{2}\right) \phi_{1}+\sum_{k=1}^{m} k A_{1, k} \phi_{2}^{k-1} .
\end{aligned}
$$

There are four auxiliary fields in eq. (3.12) which are $F_{1}, F_{1}^{*}, F_{2}, F_{2}^{*}$. The field equations obtained by varying $F_{1}, F_{1}^{*}, F_{2}, F_{2}^{*}$ are given in appendix B. These are coupled equations involving all the $F^{\prime}$ 's and $F^{*}$ 's and solving them is non-trivial. Much simplicity results if we set $\alpha_{2}=0=\alpha_{3}$. In this case as shown in appendix B the auxiliary fields $F_{k}$ satisfy the cubic equation

$$
F_{k}^{3}+p_{k} F_{k}+q_{k}=0, k=1,2,
$$

where $p_{k}, q_{k}$ are defined by

$$
\begin{aligned}
p_{k} & =\left(\frac{\partial W}{\partial \phi_{k}}\right)^{-1} \frac{\partial W^{*}}{\partial \phi_{k}^{*}} \frac{1-2 \alpha_{1} G(\phi) \partial_{\mu} \phi_{k} \partial^{\mu} \phi_{k}}{2 \alpha_{1} G(\phi)}, \\
q_{k} & =\frac{1}{2 \alpha_{1} G(\phi)}\left(\frac{\partial W}{\partial \phi_{k}}\right)^{-1}\left(\frac{\partial W^{*}}{\partial \phi_{k}^{*}}\right)^{2} .
\end{aligned}
$$


Since $F_{k}$ satisfies a cubic equation, there are three roots which are given by

$$
\begin{aligned}
F_{k}= & \omega^{j}\left(-\frac{q_{k}}{2}+\sqrt{\left(\frac{q_{k}}{2}\right)^{2}+\left(\frac{p_{k}}{3}\right)^{3}}\right)^{1 / 3} \\
& +\omega^{3-j}\left(-\frac{q_{k}}{2}-\sqrt{\left(\frac{q_{k}}{2}\right)^{2}+\left(\frac{p_{k}}{3}\right)^{3}}\right)^{1 / 3},
\end{aligned}
$$

where $\omega$ is the cube root of unity and $j=0,1,2$. Naively, it appears there are three solutions for $F_{k}$. However, as exhibited in appendix B, only $j=0$ is a solution to the full Euler-Lagrange equations for $F$. Setting the derivative terms to zero, the scalar potential of the theory for this case can be computed and is exhibited in appendix C. As an expansion in $1 / T$, the $F_{k}$ takes the form

$$
\begin{aligned}
F_{k}= & -\frac{\partial W^{*}}{\partial \phi_{k}^{*}}+\frac{1}{T}\left(\frac{\partial W}{\partial \phi_{k}}\right)\left(\frac{\partial W^{*}}{\partial \phi_{k}^{*}}\right)^{2}-\frac{3}{T^{2}}\left(\frac{\partial W}{\partial \phi_{k}}\right)^{2}\left(\frac{\partial W^{*}}{\partial \phi_{k}^{*}}\right)^{3}+\frac{12}{T^{3}}\left(\frac{\partial W}{\partial \phi_{k}}\right)^{3}\left(\frac{\partial W^{*}}{\partial \phi_{k}^{*}}\right)^{4} \\
& -\frac{55}{T^{4}}\left(\frac{\partial W}{\partial \phi_{k}}\right)^{4}\left(\frac{\partial W^{*}}{\partial \phi_{k}^{*}}\right)^{5}+\frac{273}{T^{5}}\left(\frac{\partial W}{\partial \phi_{k}}\right)^{5}\left(\frac{\partial W^{*}}{\partial \phi_{k}^{*}}\right)^{6}+\mathcal{O}\left(\frac{1}{T^{6}}\right) .
\end{aligned}
$$

Further the scalar potential when expanded in powers of $1 / T$ takes the form

$$
\begin{aligned}
V(\phi)= & \sum_{k=1}^{2}\left[\frac{\partial W}{\partial \phi_{k}} \frac{\partial W^{*}}{\partial \phi_{k}^{*}}-\frac{1}{2 T}\left(\frac{\partial W}{\partial \phi_{k}} \frac{\partial W^{*}}{\partial \phi_{k}^{*}}\right)^{2}+\frac{1}{T^{2}}\left(\frac{\partial W}{\partial \phi_{k}} \frac{\partial W^{*}}{\partial \phi_{k}^{*}}\right)^{3}\right. \\
& \left.-\frac{3}{T^{3}}\left(\frac{\partial W}{\partial \phi_{k}} \frac{\partial W^{*}}{\partial \phi_{k}^{*}}\right)^{4}+\frac{11}{T^{4}}\left(\frac{\partial W}{\partial \phi_{k}} \frac{\partial W^{*}}{\partial \phi_{k}^{*}}\right)^{5}-\frac{91}{2 T^{5}}\left(\frac{\partial W}{\partial \phi_{k}} \frac{\partial W^{*}}{\partial \phi_{k}^{*}}\right)^{6}+\mathcal{O}\left(\frac{1}{T^{6}}\right)\right] .
\end{aligned}
$$

Thus as $T \rightarrow \infty$ we recover the conventional results for $F_{k}$ and $V(\phi)$. Further, the above analysis also implies stability conditions so that

$$
\frac{\partial W}{\partial \phi_{k}}=0=\frac{\partial W^{*}}{\partial \phi_{k}^{*}}, \quad k=1,2 .
$$

We use eq. (3.18) for stabilizing the saxions. Thus we parametrize $\phi_{k}$ so that

$$
\phi_{k}=\frac{1}{\sqrt{2}}\left(f_{k}+\rho_{k}\right) e^{i a_{k} / f_{k}}, \quad k=1,2,
$$

where $\rho_{k}$ are the saxion fields, $a_{k}$ are the axions and $f_{k}$ are the axion decay constants. For the case of the assumed superpotential the stabilization conditions on a CP conserving vacuum are

$$
\begin{aligned}
& \mu f_{2}+\frac{1}{2} \lambda f_{1} f_{2}^{2}+\sum_{k=1}^{m} \frac{1}{2^{k / 2-1}} k A_{1, k} f_{1}^{k-1}=0, \\
& \mu f_{1}+\frac{1}{2} \lambda f_{1}^{2} f_{2}+\sum_{k=1}^{m} \frac{1}{2^{k / 2-1}} k A_{2, k} f_{2}^{k-1}=0 .
\end{aligned}
$$


Eqs. (3.20) and (3.21) provide the stability conditions for the radial modes $\rho_{1}$ and $\rho_{2}$. Here the first two terms on the left hand side of each of the above equations arise from $W_{s}$ while the last term involving the sum arises from $W_{s b}$. For arbitrary $m$ these equations cannot be solved in a closed form. However, we can solve for $f_{i}$ in a perturbative expansion where we treat the contribution arising from $W_{s b}$ as the perturbation. A significant simplicity results if we assume $A_{k} \equiv A_{1, k}=A_{2, k}$. In this case $f \equiv f_{1}=f_{2}$ and we find that

$$
\begin{aligned}
f & =f_{0}-\frac{2}{3 \lambda} \sum_{k} \frac{k A_{k}}{2^{k / 2-1}} f_{0}^{k-3}+\cdots \\
f_{0} & \equiv \pm \sqrt{\frac{-2 \mu}{\lambda}}
\end{aligned}
$$

Eq. (3.22) exhibits the value of the stability point $f$ of the radial modes in terms of the input parameters of the superpotential including $W_{s}$ and $W_{s b}$.

For the case of two fields, we have $F_{1}$ and $F_{2}$, which can be solved using eq. (3.15). For our choice of $W$, we can evaluate $p_{k}$ and $q_{k}(\mathrm{k}=1,2)$ explicitly so that we have

$$
\begin{aligned}
& p_{1}=\frac{\mu \phi_{2}^{*}+\lambda \phi_{1}^{*} \phi_{2}^{* 2}+\sum_{k=1}^{m} k A_{1, k} \phi_{1}^{* k-1} \frac{1-2 \alpha_{1} G(\phi) \partial_{\mu} \phi_{1} \partial^{\mu} \phi_{1}^{*}}{\mu \phi_{2}+\lambda \phi_{1} \phi_{2}^{2}+\sum_{k=1}^{m} k A_{1, k} \phi_{1}^{k-1}}}{p_{2}=\frac{\mu \phi_{1}^{*}+\lambda \phi_{1}^{* 2} \phi_{2}^{*}+\sum_{k=1}^{m} k A_{2, k} \phi_{2}^{* k-1}}{\mu \phi_{1}+\lambda \phi_{1}^{2} \phi_{2}+\sum_{k=1}^{m} k A_{2, k} \phi_{2}^{k-1}} \frac{1-2 \alpha_{1} G(\phi) \partial_{\mu} \phi_{2} \partial^{\mu} \phi_{2}^{*}}{2 \alpha_{1} G(\phi)}} \\
& q_{1}=\frac{1}{2 \alpha_{1} G\left(\phi_{1}\right)} \frac{\left(\mu \phi_{2}^{*}+\lambda \phi_{1}^{*} \phi_{2}^{* 2}+\sum_{k=1}^{m} k A_{1, k} \phi_{1}^{* k-1}\right)^{2}}{\mu \phi_{2}+\lambda \phi_{1} \phi_{2}^{2}+\sum_{k=1}^{m} k A_{1, k} \phi_{1}^{k-1}} \\
& q_{2}=\frac{1}{2 \alpha_{1} G\left(\phi_{2}\right)} \frac{\left(\mu \phi_{1}^{*}+\lambda \phi_{1}^{* 2} \phi_{2}^{*}+\sum_{k=1}^{m} k A_{2, k} \phi_{2}^{* k-1}\right)^{2}}{\mu \phi_{1}+\lambda \phi_{1}^{2} \phi_{2}+\sum_{k=1}^{m} k A_{2, k} \phi_{2}^{k-1}}
\end{aligned}
$$

In these equations we didn't impose stability conditions which must be imposed for evaluation. We now carry out a fast-roll and a slow-roll decomposition of the fields following the procedure discussed in [22] and reviewed in 2 and define

$$
\begin{aligned}
& a_{+} / f_{+}=\frac{1}{\sqrt{2}}\left(a_{1} / f_{1}+a_{2} / f_{2}\right), \\
& a_{-} / f_{-}=\frac{1}{\sqrt{2}}\left(a_{1} / f_{1}-a_{2} / f_{2}\right) .
\end{aligned}
$$

Here $a_{+}$is the field that is invariant under the shift symmetry and undergoes fast roll and $a_{-}$is the field that is sensitive to the shift symmetry and undergoes slow roll. We are interested in only the slow-roll part and thus we suppress $a_{+}$and retain only the $a_{-}$part in the potential. This topic was dealt with in great detail in [22]. Here the more general case of $m$ pairs of axion fields with each pair having fields with opposite $\mathrm{U}(1)$ charges was considered. In this case one has $2 m$ number of axionic fields. After spontaneous symmetry breaking using the superpotential $W=W_{s}$ the potential involving the $2 m$ axion fields was computed and is exhibited in eqs. (2.11) and (2.12) of [22]. This allows us to compute 
the mass matrix for the $2 m$ axion fields which is shown in eqs. (2.13)-(2.14) of [22]. Here one finds that $2 m-1$ linear combinations of axion fields gain mass and one can see that the masses of the axions are scaled by $\mu_{k l}$ which can be taken to the GUT scale. These $2 m-1$ linear combinations of axion fields are invariant under the $\mathrm{U}(1)$ global symmetry. The remaining one linear combination of axion fields corresponds to the pseudo-NambuGoldstone bosons (pNGB) and is massless. On including $W_{s b}$ in spontaneous breaking, the pNGB also gains a mass. However, this mass is much smaller than those of the other $2 m-1$ axions because $\left|W_{s b}\right| \ll\left|W_{s}\right|$. In the analysis of this paper there is just one pair of axion fields so we have only $a_{+}$and $a_{-}$where $a_{+}$is the axion field invariant under the $\mathrm{U}(1)$ global symmetry and $a_{-}$is the field which is the pNGB. In [22] it was shown that $a_{+}$ rolls an order of magnitude or more faster than $a_{-}$as shown by blue lines (fast roll) vs. red lines (slow roll) in figure 1 and figure 2 of [22]. This phenomenon also holds for the current analysis presented here.

To simplify the analysis we set $A_{1, k}=A_{2, k}=A_{k}$ and set $f_{1}=f_{2}=f_{+}=f_{-}=f$. In this case we have neglected spatial gradients:

$$
\begin{gathered}
A\left(a_{-}\right)=-\frac{\dot{a}_{-}^{2}}{2 T}, \quad B\left(a_{-}\right)=\frac{1}{8 T^{2}} \dot{a}_{-}^{4}, \\
G_{i}\left(a_{-}\right)=G\left(a_{-}\right)=\frac{1}{T-\dot{a}_{-}^{2} / 2+\sqrt{T^{2}-T \dot{a}_{-}^{2}+\frac{1}{8} \dot{a}_{-}^{4}\left(2-\alpha_{1}\right)}} .
\end{gathered}
$$

$\mathcal{L}_{I}$ is given by

$$
\mathcal{L}_{I}=T\left(1-\sqrt{1-\frac{\dot{a}_{-}^{2}}{T}+\frac{\left(2-\alpha_{1}\right) \dot{a}_{-}^{4}}{8 T^{2}}}\right) .
$$

$\mathcal{L}_{I I}$ is more complicated:

$$
\begin{aligned}
\mathcal{L}_{I I}= & T\left(2 \mathcal{F}_{+}^{2}+2 \mathcal{F}_{-}^{2}-\frac{4}{3 \alpha_{1}}\left(\mathcal{T}+\left(\alpha_{1}-1\right) \frac{\dot{a}_{-}^{2}}{4 T}\right)+4 k\left(\mathcal{F}_{+}+\mathcal{F}_{-}\right)\right. \\
& +\frac{\alpha_{1}}{\mathcal{T}-\dot{a}_{-}^{2} /(4 T)}\left(2\left(\mathcal{F}_{+}^{2}+\mathcal{F}_{-}^{2}-\frac{2}{3 \alpha_{1}}\left(\mathcal{T}+\left(\alpha_{1}-1\right) \frac{\dot{a}_{-}^{2}}{4 T}\right)\right) \frac{\dot{a}_{-}^{2}}{4 T}+\mathcal{F}_{+}^{4}+\mathcal{F}_{-}^{4}\right. \\
& \left.\left.+\frac{2}{3 \alpha_{1}^{2}}\left(\mathcal{T}+\left(\alpha_{1}-1\right) \frac{\dot{a}_{-}^{2}}{4 T}\right)^{2}-\frac{4}{3 \alpha_{1}}\left(\mathcal{T}+\left(\alpha_{1}-1\right) \frac{\dot{a}_{-}^{2}}{4 T}\right)\left(\mathcal{F}_{+}^{2}+\mathcal{F}_{-}^{2}\right)\right)\right)
\end{aligned}
$$

where

$$
\begin{aligned}
& \mathcal{T}=\frac{1}{2}\left(1+\sqrt{1-\frac{\dot{a}_{-}^{2}}{T}+\frac{\left(2-\alpha_{1}\right) \dot{a}_{-}^{4}}{8 T^{2}}}\right), \\
& k=\tilde{\beta} \sqrt{\sum_{m, n} m n \mathcal{G}_{m} \mathcal{G}_{n}\left(1-\cos \left(\frac{a_{-} m}{\sqrt{2} f}\right)-\cos \left(\frac{a_{-} n}{\sqrt{2} f}\right)+\cos \left(\frac{a_{-}(m-n)}{\sqrt{2} f}\right)\right)},
\end{aligned}
$$




$$
\begin{aligned}
& \mathcal{F}_{ \pm}= \pm\left(\mp \frac{1}{2 \alpha_{1}} k\left(\mathcal{T}-\frac{\dot{a}_{-}^{2}}{4 T}\right)+\sqrt{\frac{1}{4 \alpha_{1}^{2}} k^{2}\left(\mathcal{T}-\frac{\dot{a}_{-}^{2}}{4 T}\right)^{2}+\frac{1}{27 \alpha_{1}^{3}}\left(\mathcal{T}+\left(\alpha_{1}-1\right) \frac{\dot{a}_{-}^{2}}{4 T}\right)^{3}}\right)^{1 / 3}, \\
& \mathcal{G}_{k}=\frac{A_{k} 2^{1 / 2(1-k)}}{\tilde{\beta} \sqrt{T} f^{1-k}} .
\end{aligned}
$$

Here $\tilde{\beta}$ is an arbitrary dimensionless parameter which we choose such that $\mathcal{G}_{k} \sim 1$, and which determines the scale of symmetry breaking terms relative to $T$.

Note that there are two ways here to achieve $A_{k} \ll \mu \approx M_{\mathrm{P}}$, which is the condition needed for slow-roll approximation. One way is to consider small values of $\tilde{\beta}$, in which case we found DBI plays insignificant role and we reproduce the results of [22]. Another way is to consider $T \ll M_{\mathrm{P}}$, and $\tilde{\beta} \approx M_{\mathrm{P}}$, which is the case we will focus on for the remainder of this paper.

\section{Pressure, density and inflation equations}

The pressure $p$ and density $\rho$ are defined in terms of the stress tensor by

$$
p=\frac{1}{3} \sum_{i=1}^{3} T^{i i}, \quad \rho=T^{00},
$$

where $T^{\mu \nu}(\mu=0,1,2,3)$ is the stress tensor and is given by

$$
T^{\mu \nu}=g^{\mu \nu} \mathcal{L}\left(\phi_{k}, \partial_{\mu} \phi_{k} \partial^{\mu} \phi_{k}\right)-2 \frac{\delta \mathcal{L}\left(\phi_{k}, \partial_{\mu} \phi_{k} \partial^{\mu} \phi_{k}\right)}{\delta\left(\partial_{\mu} \phi_{k} \partial^{\mu} \phi_{k}\right)} \partial^{\mu} \phi_{k} \partial^{\nu} \phi_{k},
$$

and we use the metric $\eta^{\mu \nu}=\operatorname{diag}(-1,1,1,1)$. In the analysis we assume space to be homogeneous and isotropic, so that $\partial_{i} \phi_{k}=0$ for $1 \leq i \leq 3$ and $\partial_{0} \phi_{k}=\dot{\phi}_{k}$, one finds that eqs. (4.1) and (4.2) for pressure and density become:

$$
p=\mathcal{L}\left(\phi_{k},-\beta_{k}\right),
$$

and

$$
\rho=-\mathcal{L}\left(\phi_{k},-\beta_{k}\right)+2 \sum_{k} \frac{\delta \mathcal{L}\left(\phi_{k},-\beta_{k}\right)}{\delta \beta_{k}} \beta_{k},
$$

where $\beta_{k}=\dot{\phi}_{k}^{2}$. These relations are valid for non-canonical kinetic terms. The Friedman equations are given by

$$
\begin{aligned}
\dot{\rho} & =-3 H(p+\rho), \\
3 M_{\mathrm{P}}^{2} \frac{\dot{R}^{2}}{R^{2}} & =\rho, \\
\dot{\rho} & =-6 H \sum_{k} \frac{\partial L}{\partial \beta_{k}} \beta_{k} .
\end{aligned}
$$


Further taking the time derivative of eq. (4.4) which along with eq. (4.7) gives

$$
\sum_{k}\left[\left(2 \frac{\partial^{2} L}{\partial \beta_{k} \partial \beta_{k}} \beta_{k}+\frac{\partial L}{\partial \beta_{k}}\right) \dot{\beta}_{k}-\frac{\partial L}{\partial \phi_{k}} \dot{\phi}_{k}+6 H \frac{\partial L}{\partial \beta_{k}} \beta_{k}+2 \frac{\partial^{2} L}{\partial \phi_{k} \partial \beta_{k}} \beta \dot{\phi}_{k}\right]=0 .
$$

Next we focus on the slow-roll part where we keep only the field $a_{-}$. In this case eqs. (4.6) and (4.8) can be written in the following form

$$
\begin{array}{r}
3 M_{\mathrm{P}}^{2} \frac{\dot{R}^{2}}{R^{2}}=2 \dot{a}_{-}^{2} \frac{\partial L}{\partial \dot{a}_{-}^{2}}-L, \\
2\left[2 \frac{\partial^{2} L}{\partial \dot{a}_{-}^{2} \partial \dot{a}_{-}^{2}} \dot{a}_{-}^{2}+\frac{\partial L}{\partial \dot{a}_{-}^{2}}\right] \ddot{a}_{-}-\frac{\partial L}{\partial a_{-}}+6 H \dot{a}_{-} \frac{\partial L}{\partial \dot{a}_{-}^{2}}+2 \frac{\partial^{2} L}{\partial a_{-} \partial \dot{a}_{-}^{2}} \dot{a}_{-}^{2}=0 .
\end{array}
$$

For the case of canonical kinetic energy and no dependence of the potential on time derivative of field, i.e.,

$$
L=-\frac{1}{2} \partial_{\mu} a_{-} \partial a_{-}^{\mu}-V\left(a_{-}\right) .
$$

Eqs. (4.9) and (4.10) reduce to the following

$$
\begin{aligned}
3 M_{\mathrm{P}}^{2} \frac{\dot{R}^{2}}{R^{2}} & =\frac{1}{2} \dot{a}_{-}^{2}+V\left(a_{-}\right), \\
\ddot{a}_{-}+3 H \dot{a}_{-}+V^{\prime}\left(a_{-}\right) & =0,
\end{aligned}
$$

which are correctly the relation for slow roll for the case when one has canonical kinetic energy and the potential is velocity independent. However, in our case we have more terms. Here we emphasize that while for conventional inflation models the potential plays a central role in the analysis, this is not the case for the Dirac-Born-Infeld case. Here the potential by itself is not sufficient and the entire Lagrangian enters in the analysis. The potential in isolation plays no role. This can be seen from the Friedman equations eqs. (4.9) and (4.10) above where the full Lagrangian and not just the potential enters. Only in the case when we assume canonical kinetic energy and no dependence of the potential on the derivatives of the fields that one recovers the conventional Friedman equations where the potential appears as shown in eqs. (4.12).

\section{Slow roll parameters and non-Gaussianity}

For non-canonical kinetic energy terms and for velocity dependent potential a quantity that enters the analysis of slow roll parameters is the speed of sound $c_{s}$ defined by

$$
c_{s}^{2}=\frac{p, \beta}{\rho, \beta} .
$$

$c_{s}$ also enters in the analysis of non-Gaussianity to be discussed later. The speed of sound is limited by the constraint $0<c_{s}^{2} \leq 1$. Often a parameter $\gamma$ is used which is defined by $\gamma=\frac{1}{c_{s}}$ and lies in the range $1 \leq \gamma<\infty$. For models with canonical kinetic energy $\gamma=1$ 
and in this case there is no non-Gaussianity. For non-Gaussianity one requires $\gamma>1$. For the models we consider one may write $\gamma^{2}$ as follows

$$
\gamma^{2}=1+2 \dot{a}_{-}^{2} \frac{\partial^{2} L}{\partial \dot{a}_{-}^{2} \partial \dot{a}_{-}^{2}} / \frac{\partial L}{\partial \dot{a}_{-}^{2}}
$$

and eq. (4.10) can then be written as follows

$$
\frac{\partial L}{\partial\left(\dot{a}_{-}^{2} / 2\right)} \ddot{a}_{-}-\frac{1}{\gamma^{2}} \frac{\partial L}{\partial a_{-}}+\frac{3}{\gamma^{2}} H \dot{a}_{-} \frac{\partial L}{\partial\left(\dot{a}_{-}^{2} / 2\right)}+\frac{1}{\gamma^{2}} \frac{\partial^{2} L}{\partial a_{-} \partial\left(\dot{a}_{-}^{2} / 2\right)} \dot{a}_{-}^{2}=0 .
$$

The first three terms on the left hand side are similar to what one has normally except for $\gamma$ dependence. The last term on the left hand side is new.

We define the slow roll parameters for DBI as [24-29]

$$
\epsilon=-\frac{\dot{H}}{H^{2}}, \quad \eta=\frac{\dot{\epsilon}}{\epsilon H}, \quad s=\frac{\dot{c}_{s}}{c_{s} H} .
$$

In terms of these parameters the power spectrum for the scalar perturbations $P_{k}^{\zeta}$ and the power spectrum for the tensor perturbations $P_{k}^{h}$ are given by [61, 62]:

$$
\begin{aligned}
P_{k}^{\zeta} & =\frac{1}{8 \pi^{2} M_{\mathrm{P}}^{2}} \frac{H^{2}}{c_{s} \epsilon}, \\
P_{k}^{h} & =\frac{2}{3 \pi^{2}} \frac{\rho}{M_{\mathrm{P}}^{4}} .
\end{aligned}
$$

Further, the spectral indices $n_{s}$ and $n_{t}$ in this case are given by

$$
\begin{aligned}
& n_{s}=1-2 \epsilon-\eta-s, \\
& n_{t}=-2 \epsilon,
\end{aligned}
$$

and the ratio $r$ of the tensor to the scalar power spectrum is [61]

$$
r=\frac{P_{k}^{h}}{P_{k}^{\zeta}}=-8 c_{s} n_{t}
$$

One may compare it to the conventional slow-roll parameters $\epsilon_{V}, \eta_{V}$ for the case of the canonical kinetic energy term and no velocity dependence in the potential. Here one defines $\epsilon_{V}, \eta_{V}$ so that

$$
\begin{aligned}
\epsilon_{V} & =\frac{M_{\mathrm{P}}^{2}}{2}\left(\frac{V^{\prime}}{V}\right)^{2}, \\
\eta_{V} & =M_{\mathrm{P}}^{2} \frac{V^{\prime \prime}}{V},
\end{aligned}
$$

and the spectral indices and the ratio of the tensor and the scalar power spectrum in this case are given by

$$
n_{s}=1-6 \epsilon_{V}+2 \eta_{V}, \quad n_{t}=-2 \epsilon_{V}, \quad r=16 \epsilon_{V} .
$$


To establish a connection between the DBI slow-roll parameters $\epsilon, \eta$ with the conventional slow-roll parameters we note that in the conventional slow roll one assumes dominance of the potential and one sets $c_{s}=1$ and makes the following approximations

$$
\dot{\phi} \simeq-\frac{V^{\prime}}{3 H}, \quad H^{2} \simeq \frac{V}{3 M_{\mathrm{P}}^{2}}, \quad H^{\prime}=\frac{V^{\prime}}{6 M_{\mathrm{P}}^{2} H} .
$$

Using these one can connect $\epsilon, \eta$ to $\epsilon_{V}, \eta_{V}$ so that

$$
\begin{aligned}
\epsilon & =\epsilon_{V}, \\
\eta & =-2 \eta_{V}+4 \epsilon_{V} .
\end{aligned}
$$

Using $c_{s}=1$ and eq. (5.11) in eqs. (5.6) and (5.7) we can recover eq. (5.9).

Non-Gaussianity is defined by the three-point correlation function of perturbations involving three scalars, two scalars and a graviton, two gravitons and a scalar and three gravitons [24]. Since the work of [24] there has been a significant number of further analyses (see, e.g., [25, 27-30, 32, 34, 63-68]). The dominant non-Gaussianity arises from the correlation function of three scalar perturbations. Thus for scalar perturbation $\zeta(\vec{k})$ nonGaussianity is defined by

$$
\left\langle\zeta\left(\vec{k}_{1}\right) \zeta\left(\vec{k}_{2}\right) \zeta\left(\vec{k}_{3}\right)\right\rangle=(2 \pi)^{7} \delta^{3}\left(\vec{k}_{1}+\vec{k}_{2}+\vec{k}_{3}\right) \frac{\sum_{i} k_{i}^{3}}{\prod_{i} k_{i}^{3}}\left[-\frac{3}{10} f_{\mathrm{NL}}\left(P_{k}^{\zeta}\right)^{2}\right],
$$

where $P_{k}^{\zeta}$ is the scalar power spectrum and $f_{\mathrm{NL}}$ is a measure of non-Gaussianity. NonGaussianity depends strongly on shape and various types of shapes have been discussed in the literature which include local, equilibrium and orthogonal [69] (for earlier analyses see [70-72]). The non-Gaussianity we will discuss here is the one which has the shape of an equilateral triangle, when $k_{1}=k_{2}=k_{3}$ and is given by [28]

$$
f_{\mathrm{NL}}^{\text {equil }}=\frac{35}{108}\left(\frac{1}{c_{s}^{2}}-1\right)-\frac{5}{81}\left[\left(\frac{1}{c_{s}^{2}}-1-2 \frac{z_{2}}{z_{1}}\right)+\left(3-2 c_{1}\right) z \frac{z_{2}}{z_{1}}\right]+\mathcal{O}(\epsilon),
$$

where

$$
z_{1}=\beta L_{, \beta}+2 \beta^{2} L_{\beta \beta}, \quad z_{2}=\beta^{2} L_{, \beta \beta}+\frac{2}{3} \beta^{3} L_{, \beta \beta \beta}, \quad z=\frac{\dot{z}_{2}}{z_{2} H}
$$

where the superscript "equil" on $f_{\mathrm{NL}}$ indicates that we are specifically considering the shape to be of an equilateral triangle.

\section{Model simulation and experimental test}

Numerical simulations performed consist of two parts: a large $0.5 \times 10^{6}$ points simulation in which $\alpha_{2}=\alpha_{3}=0$, and a small simulation with $\alpha_{2}$ and $\alpha_{3}$ perturbed around an experimentally consistent point with the largest non-Gaussianity from the first simulation. The evolution of the inflaton field $a_{-}$in the large simulation of figures 1,2 , and 4 is obtained by taking the effective axion Lagrangian eqs. (3.30) and (3.31), deriving Euler-Lagrange equation and Friedmann eq. (4.6) using computer algebra, and solving them numerically. 

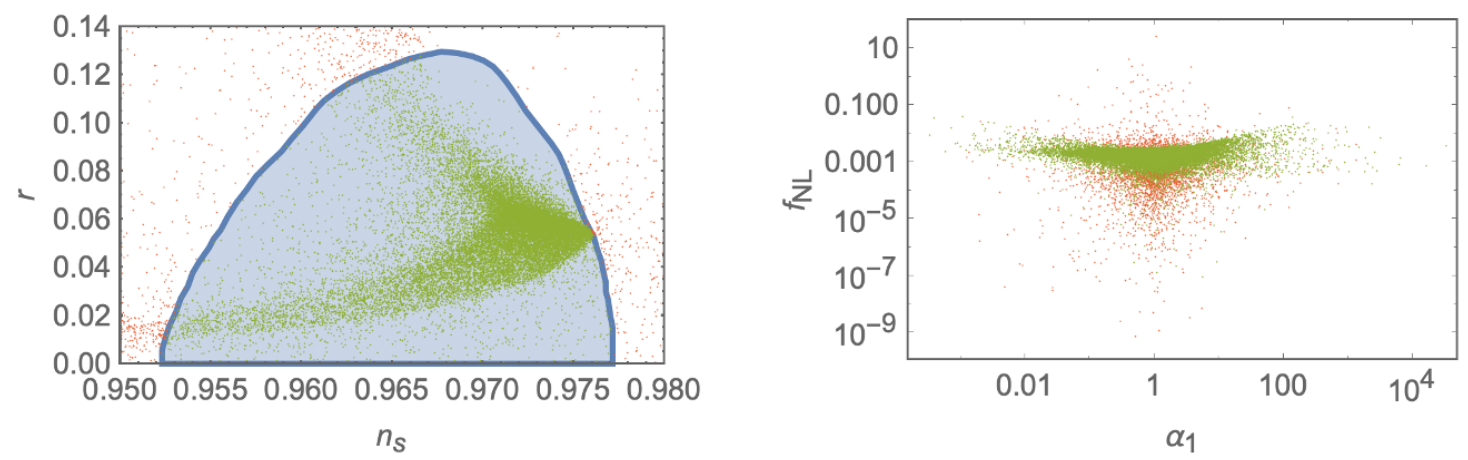

Figure 1. Left panel: a plot of the ratio $r$ of the tensor to scalar power spectrum vs. the scalar spectral index $n_{s}$ for the two field DBI model. The blue region enclosed by the blue line is the one allowed by experiment in the $2 \sigma$ range. $85 \%$ of the simulated points are consistent with Planck constraints as shown by green points while the red points lie outside the $68 \%$ CL contour. Right panel: a plot of non-Gaussianity parameter $f_{\mathrm{NL}}^{\text {equil }}$ as a function of $\alpha_{1}$ for the same data set as in the left plot. As in the left panel the green dots are parameter points which satisfy the Planck constraints on $r$ and $n_{s}$ and lie in the blue region and the red dots are parameter points which are outside the experimentally allowed region on $r$ and $n_{s}$. Points are distributed according to the following: $\alpha_{1} \sim X_{0, \infty}, \alpha_{2}=\alpha_{3}=0, T=10^{-12} M_{\mathrm{P}}^{4}, f \sim X_{0, \infty} M_{\mathrm{P}}, \tilde{\beta} \sim X_{0, \infty}, m=6$, $\mathcal{G}_{1}=\mathcal{G}_{2}=\mathcal{G}_{3}=0, \mathcal{G}_{4}=1, \mathcal{G}_{5} \sim \mathcal{G}_{6} \sim \mathcal{U}\{-1,1\} \times X_{0, \infty}, N_{\text {pivot }} \sim \mathcal{U}(50,60), a_{-, 0} \sim \mathcal{U}(0,2 \pi) \times f$, $\dot{a}_{-, 0} \sim \mathcal{U}(-1,1) \times\left(2 \sqrt{T} /\left(\sqrt{2} \sqrt{\alpha_{1}}+2\right)^{1 / 2}\right)$ where $X_{0, \infty}=\mathcal{U}\left\{X_{0,1}, 1 / X_{0,1}\right\}, X_{0,1}=\mathcal{U}(0,1), \mathcal{U}$ refers to a uniform distribution, and the distribution of $\dot{a}_{-, 0}$ is chosen such that the expression under the square root in eq. (3.30) is positive. Points are further filtered such that the mass of the inflaton $m_{a_{-}}<0.1 M_{\mathrm{P}}$.
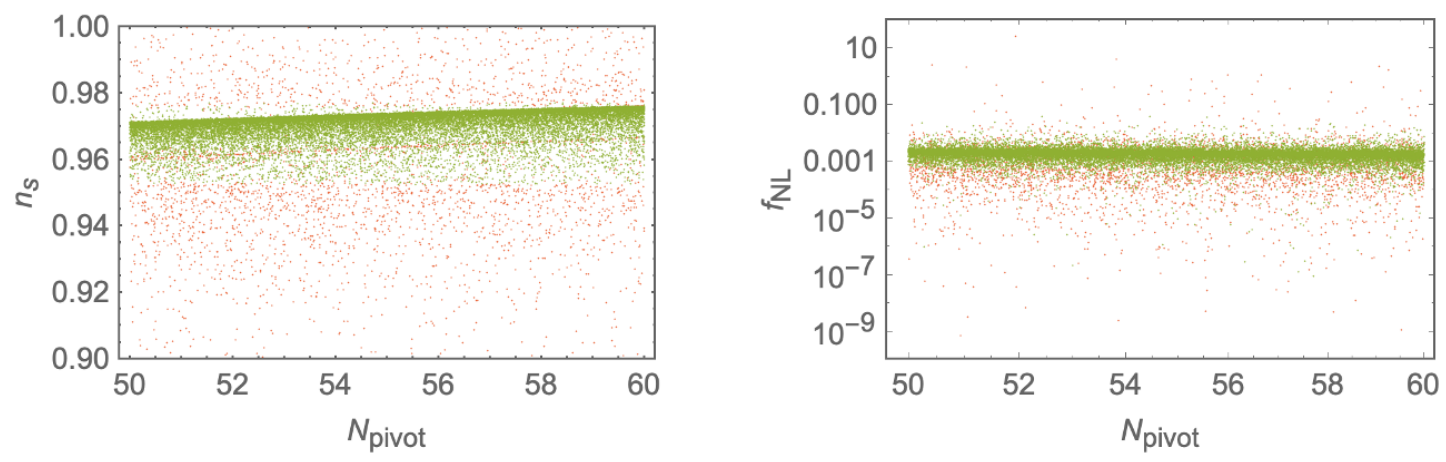

Figure 2. Left panel: a plot of the ratio $n_{s}$ vs. $N_{\text {pivot }}$ in the range 50-60 for the same data set as in figure 1. The plot shows a mild dependence of $n_{s}$ on $N_{\text {pivot }}$ in the range indicated. Right panel: the same as the left panel except $f_{\mathrm{NL}}^{\text {equil }}$ is plotted vs. $N_{\text {pivot }}$. The green and red dots are parameter points and have the same meaning as in figure 1 . 

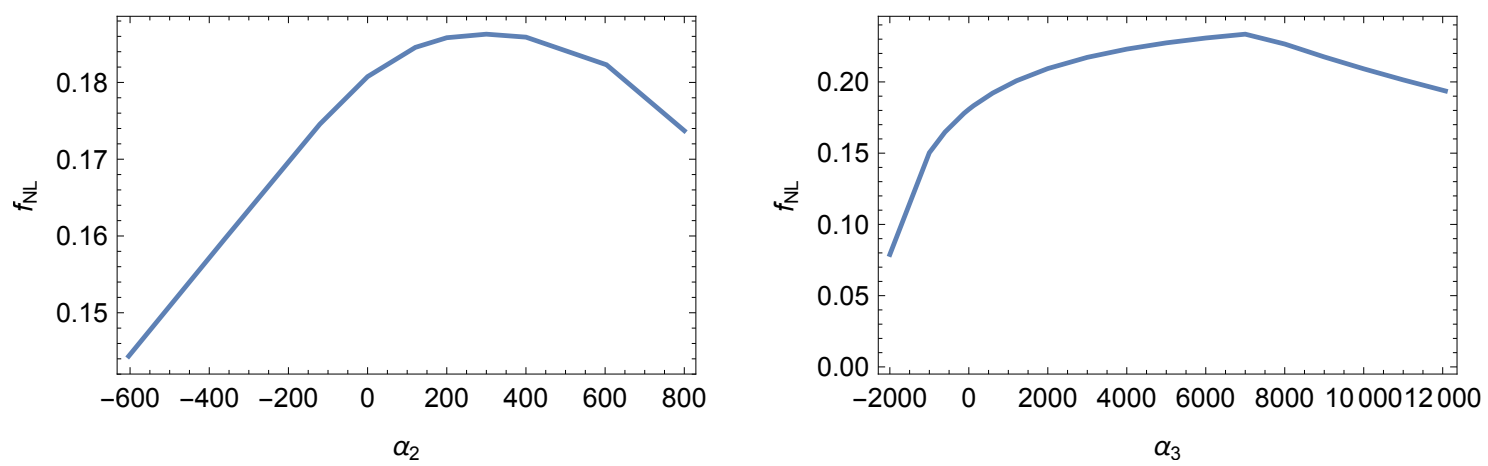

Figure 3. The effect of $\alpha_{2}$ (left panel) and $\alpha_{3}$ (right panel) on non-Gaussianity for a parameter point taken from figure 1 which is consistent with Planck data and has the largest non-Gaussianity among the set of parameter points in figure 1. Specifically, here $f=0.429 M_{\mathrm{P}}, a_{-, 0}=0.233 M_{\mathrm{P}}$, $\dot{a}_{-, 0}=-0.118 \sqrt{T}, m=6, \mathcal{G}_{1}=\mathcal{G}_{2}=\mathcal{G}_{3}=0, \mathcal{G}_{4}=1, \mathcal{G}_{5}=-1.53, \mathcal{G}_{6}=563, \alpha_{1}=1207, \tilde{\beta}=0.802$, $T=10^{-12} M_{\mathrm{P}}^{4}$. One finds that the effect is not sufficient to obtain a significantly larger value of $f_{\mathrm{NL}}$.

The integration is stopped when $\ddot{a}=0$ where $a$ is the scale factor and one may write the end of inflation constraint as

$$
\ddot{a}=a H^{2}\left(1+\frac{\dot{H}}{H^{2}}\right)=0 .
$$

An analytic expression similar to eq. (3.31), however, cannot be derived for non-zero $\alpha_{2}$ and $\alpha_{3}$. In this case, equations for the auxiliary fields $F_{k}$ are solved numerically, and the Lagrangian in terms of $\phi$ (eq. (3.12)) is also evaluated numerically for each set of values for $\left(a_{-}, \dot{a}_{-}\right)$. This process is significantly slower, therefore, in this case the simulations are only done for 29 sets of parameter values as can be seen in figure 3 . The time of horizon exit is then found by counting $N_{\text {pivot }}$ e-foldings back from the end of integration, where $N_{\text {pivot }}$ is varied between 50 and 60 .

Finally, we evaluate the experimental observables at the time of horizon exit using equations from section 5 and reusing Euler-Lagrange and Friedmann equations to compute higher time derivatives of the Lagrangian, pressure and density. The observables computed are the ratio $r$ of the power spectrum of tensor to scalar perturbations, the spectral indices of scalar $n_{s}$ and tensor $n_{t}$ perturbations, speed of sound $c_{s}$ and the non-Gaussianity amplitude $f_{\mathrm{NL}}$. In the $0.5 \times 10^{6}$ points Monte Carlo analysis we allow $\alpha_{1}$ to vary (full distribution is specified in figure 1) and search for solutions that satisfy the experimental constraints. Thus the current experimental limits from Planck experiment at $k_{0}=0.05 \mathrm{Mpc}^{-1}$ are as follows $[13-15]$

$$
\begin{aligned}
n_{s} & =0.9645 \pm 0.0049(68 \% \mathrm{CL}), \\
r & <0.07(95 \% \mathrm{CL}) .
\end{aligned}
$$

and the current experimental constraint on $c_{s}$ is [14]

$$
c_{s} \geq 0.087 \quad(\text { at } 95 \% C L) .
$$



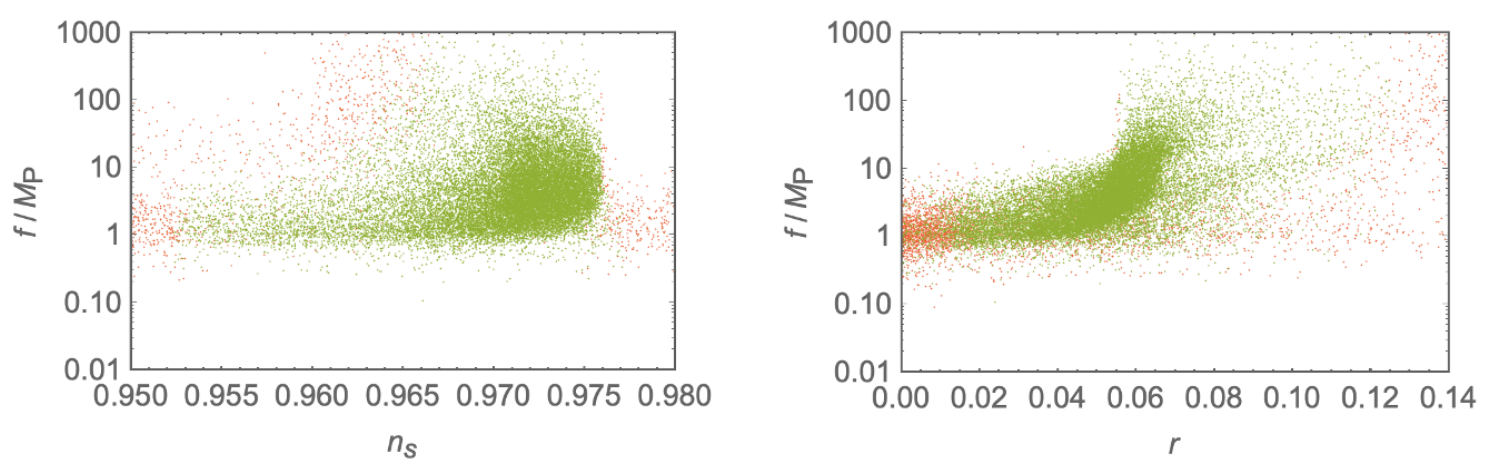

Figure 4. Left panel: a plot of $f / M_{\mathrm{P}}$ as a function of $n_{s}$ which is in the allowed range of experiment and $N_{\text {pivot }}$ in the range 50-60 for the same data set as in figure 1. Right panel: the same as the left panel except that the plot is of $f / M_{\mathrm{P}}$ as a function of $r$. We note that there are experimentally consistent points with $f / M_{\mathrm{P}} \in[0.1,1]$.

The results of the analysis for the two field supersymmetric DBI discussed above are presented in figures 1,2 , and 4 . The left panel of figure 1 gives a plot of $r$ vs. $n_{s}$. Here the region enclosed by the blue line is the one allowed by experiment in the $2 \sigma$ range. The Monte Carlo analysis shows that the experimentally allowed region is well populated by the parameter points of the model. One interesting feature of the analysis is that compared to the supersymmetric axion models discussed in [22] where $r$ was extremely small, here $r$ has significantly larger values. Thus the largeness of $r$ discriminates this class of supersymmetric DBI models from the models of [22]. The right panel of figure 1 gives a plot of $f_{\mathrm{NL}}^{\text {equil }}$ vs. $\alpha_{1}$. We see a significant sensitivity of $f_{\mathrm{NL}}^{\text {equil }}$ to $\alpha_{1}$. However, overall $f_{\mathrm{NL}}^{\text {equil }}$ is typically small and mostly lies below 0.5. The effect of $\alpha_{2}$ and $\alpha_{3}$ for the point from figure 1 with largest non-Gaussianity is shown on figure 3. One can see that although non-Gaussianity $f_{\mathrm{NL}}$ is sensitive to $\alpha_{2}$ and $\alpha_{3}$, it still lies below 0.5. This level of non-Gaussianity appears too low for observation in the near future.

The most recent analyses of non-Gaussianity based on Planck data are given in [69]. Here the analysis is done using cosmic microwave background (CMB) temperature and E-mode polarization data. The analysis using temperature alone give $f^{\text {equil }}=-16 \pm 70$ (68\% CL, statistical) and an analysis combining temperature and polarization data gives $f^{\text {equil }}=-4 \pm 43(68 \% \mathrm{CL}$, statistical $)$. In the left panel of figure 2 a plot of $n_{s}$ vs. $N_{\text {pivot }}$ is given. Here one finds that $n_{s}$ has a mild positive slope as a function of $N_{\text {pivot }}$. The right panel gives a plot of $f_{\mathrm{NL}}^{\text {equil }}$ on $N_{\text {pivot }}$. Here one finds the $f_{\mathrm{NL}}^{\text {equil }}$ has a relatively small variation in the range $[50,60]$ for $N_{\text {pivot }}$. In either case one cannot draw any significant conclusion regarding the dependence of these parameters on the number of e-foldings as long as one is in the $N_{\text {pivot }}$ range of $[50,60]$.

Then, in figure 4 we exhibit that the analysis contains a significant part of the parameter space where inflation consistent with the desired number of e-foldings in the range $[50,60]$ and $n_{s}$ and $r$ consistent with Planck data can be gotten in the axion inflation model with $f / M_{\mathrm{P}}<1$. Thus the left panel of figure 4 exhibits $f / M_{\mathrm{P}}$ as a function of $n_{s}$ in the range allowed by the Planck data constraint of eq. (6.2) and the right panel of figure 4 

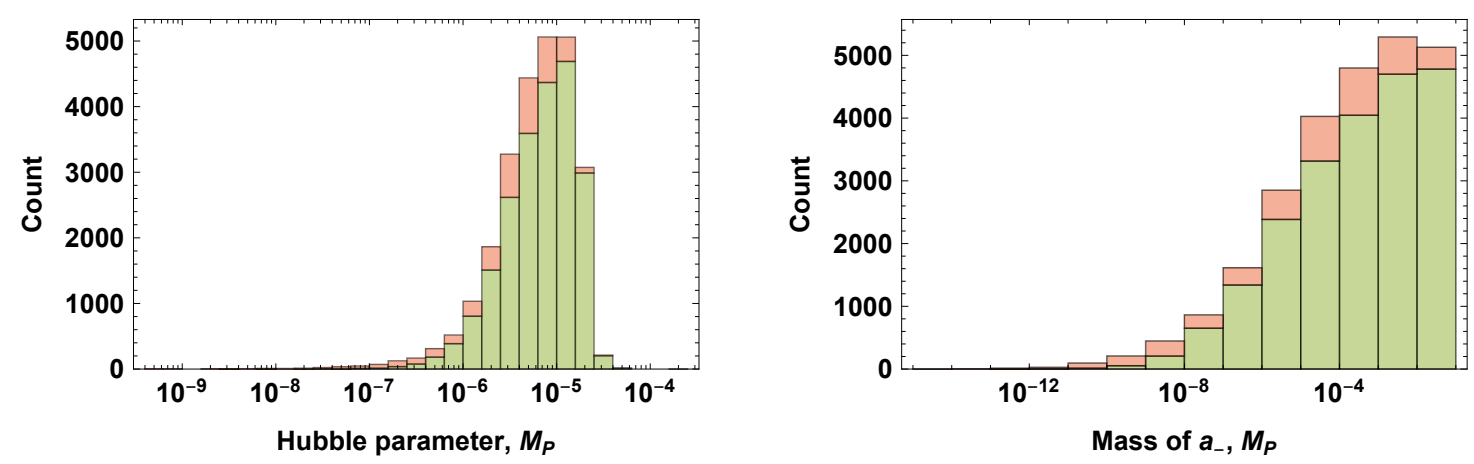

Figure 5. Left panel: a histogram of the values of Hubble parameter at horizon exit for the same data set as in figure 1 for points that are consistent (green) and not consistent (red) with experimental constraints on $r$ and $n_{s}$. Right panel: the same as the left panel except the histogram of the inflaton $a_{-}$mass is shown instead.

gives $f / M_{\mathrm{P}}$ as a function of $r$ containing the range in $r$ consistent with the Planck data constraint of eq. (6.2).

Finally, we demonstrate in figure 5 that both the Hubble parameter at horizon exit, and mass of the inflaton $a_{-}$are much smaller than $M_{\mathrm{P}}$, which are necessary and sufficient conditions for the slow-roll approximation to hold.

\section{Conclusion}

In this work we have analyzed inflation in a supersymmetric Dirac-Born-Infeld action with a U(1) symmetry. Specifically we have carried out a detailed analysis of a pair of chiral DBI fields which possess opposite charges under the U(1) symmetry. A transformation is then made to go to the co-ordinate frame where a linear combination of the axion fields is invariant under the global $\mathrm{U}(1)$ transformations and an orthogonal combination is variant which acts as the inflaton. The $\mathrm{U}(1)$ shift symmetry is broken by instanton type nonperturbative terms in the superpotential. The analysis is done in the vacuum state with stabilized saxions and the scalar potential can be decomposed into a fast-roll and a slowroll part where the slow-roll part of the potential is now velocity dependent. This velocity dependence is a direct consequence of the DBI form of the action. In the analysis for the case $\alpha_{2}=0=\alpha_{3}$ we have obtained an explicit form for the auxiliary fields $F_{k}$ which satisfy a cubic equation in terms of the inflation field. We have analyzed the scalar and tensor power spectrum and computed the spectral indices. It is shown that a significant part of the parameter space exists which supports inflation consistent with the current experimental constraints on the ratio of the tensor to the scalar power spectrum and the spectral indices. A remarkable aspect of the proposed supersymmetric DBI model is that the model supports an observable value of the tensor to the scalar power spectrum and consequently also a significant value for the spectral index $n_{t}$. This is in contrast to a supersymmetric non-DBI model which typically has a suppressed value of $r$ and of $n_{t}$. An analysis of non-Gaussianity in the model was also carried out. It is found that for the model 
parameters that support inflation consistent with the Planck experimental values on $r$ and $n_{s}$, the non-Gaussianity is typically small. This holds true even when the parameters $\alpha_{2}$ and $\alpha_{3}$ along with $\alpha_{1}$ are included in the analysis. It is of interest to achieve this class of models in string theory using moduli stabilization of the type used in KKLT [73] or the Large Volume Scenario [74].

\section{Acknowledgments}

This research was supported in part by the NSF Grant PHY-1620575.

\section{A Single field supersymmetric DBI Lagrangian}

To define notation and to discuss the technique used in the analysis of this work we consider the case here of a single superfield. Thus we consider a supersymmetric DBI Lagrangian of the form $[54,55,58]$

$$
\mathcal{L}_{\mathrm{DBI}}=\int d^{4} \theta\left(\Phi \Phi^{\dagger}+\frac{1}{16}\left(D^{\alpha} \Phi D_{\alpha} \Phi\right)\left(\bar{D}^{\dot{\alpha}} \Phi^{\dagger} \bar{D}_{\dot{\alpha}} \Phi^{\dagger}\right) G(\Phi)\right),
$$

where $\Phi$ and $\Phi^{\dagger}$ are the chiral and anti-chiral superfields, $D_{\alpha}$ and $\bar{D}_{\dot{\alpha}}$ are the supercovariant derivatives, and $G(\Phi)$ is given by

$$
G(\Phi)=\frac{1}{T} \frac{1}{1+A(\Phi)+\sqrt{(1+A(\Phi))^{2}-B(\Phi)}} .
$$

Here $T$ is a parameter of the dimension of (mass) $)^{4}$ and is related to the warp factor from the point of view of reduction of a ten dimensional theory to 4 dimensions. $A$ and $B$ are given by

$$
A(\Phi)=\frac{\partial_{\mu} \Phi \partial^{\mu} \Phi^{\dagger}}{T}, \quad B(\Phi)=\frac{\partial_{\mu} \Phi \partial^{\mu} \Phi \partial_{\nu} \Phi^{\dagger} \partial^{\nu} \Phi^{\dagger}}{T^{2}} .
$$

Ignoring fermions, the superfields have the expansion:

$$
\begin{aligned}
\Phi(x, \theta, \bar{\theta}) & =\Phi_{L}(x, \theta, \bar{\theta}) \\
& =\phi(x)+\theta^{\alpha} \theta_{\alpha} F(x)+i \theta^{\alpha} \sigma_{\alpha \dot{\alpha}}^{\mu} \bar{\theta}^{\dot{\alpha}} \partial_{\mu} \phi(x)+\frac{1}{4} \theta^{\alpha} \theta_{\alpha} \bar{\theta}_{\dot{\alpha}} \bar{\theta}^{\dot{\alpha}} \partial^{\mu} \partial_{\mu} \phi(x), \\
\Phi^{\dagger}(x, \theta, \bar{\theta}) & =\Phi_{R}(x, \theta, \bar{\theta}) \\
& =\phi^{*}(x)+\bar{\theta}_{\dot{\alpha}} \bar{\theta}^{\dot{\alpha}} F^{*}(x)-i \theta^{\alpha} \sigma_{\alpha \dot{\alpha}}^{\mu} \bar{\theta}^{\dot{\alpha}} \partial_{\mu} \phi^{*}(x)+\frac{1}{4} \theta^{\alpha} \theta_{\alpha} \bar{\theta}_{\dot{\alpha}} \bar{\theta}^{\dot{\alpha}} \partial^{\mu} \partial_{\mu} \phi^{*}(x) .
\end{aligned}
$$

$\Phi \Phi^{\dagger}$ can be written in the form

$$
\begin{aligned}
\Phi \Phi^{\dagger}= & \phi \phi^{*}+\theta^{2} \phi^{*} F+\bar{\theta}^{2} \phi F^{*}+i \theta \sigma^{\mu} \bar{\theta}\left(\phi^{*} \partial_{\mu} \phi-\phi \partial_{\mu} \phi^{*}\right) \\
& +\theta^{2} \bar{\theta}^{2}\left(F F^{*}+\frac{1}{4} \phi \partial^{\mu} \partial_{\mu} \phi^{*}+\frac{1}{4} \phi^{*} \partial^{\mu} \partial_{\mu} \phi-\frac{1}{2} \partial^{\mu} \phi^{*} \partial_{\mu} \phi\right)
\end{aligned}
$$


Further, since $\int d^{4} \theta \Phi \Phi^{\dagger}$ is the additive term in the Lagrangian, we can integrate by parts and get

$$
\int d^{2} \theta d^{2} \bar{\theta} \Phi \Phi^{\dagger}=F F^{*}-\partial^{\mu} \phi^{*} \partial_{\mu} \phi
$$

Next we note that

$$
D_{\beta} \Phi=\left(\partial_{\beta}+i \sigma_{\beta \dot{\beta}}^{\mu} \bar{\theta}^{\dot{\beta}} \partial_{\mu}\right) \Phi
$$

This leads to

$$
D_{\beta} \Phi=-2 \theta_{\beta} F(x)+2 i \sigma_{\beta \dot{\gamma}}^{\kappa} \bar{\theta}^{\dot{\gamma}} \partial_{\kappa} \phi(x)+i \theta^{\gamma} \theta_{\gamma} \sigma_{\beta \dot{\beta}}^{\mu} \bar{\theta}^{\dot{\beta}} \partial_{\mu} F(x) .
$$

Using the above we can compute $D^{\alpha} \Phi D_{\alpha} \Phi$ and the computation gives

$$
D^{\alpha} \Phi D_{\alpha} \Phi=4 F^{2} \theta^{2}-4 \partial^{\kappa} \phi \partial_{\kappa} \phi \bar{\theta}^{2}-8 i F \partial_{\kappa} \phi \theta \sigma^{\kappa} \bar{\theta}-4 \partial^{\mu} F \partial_{\mu} \phi \theta^{2} \bar{\theta}^{2} .
$$

The conjugate of eq. (A.10) can be computed as follows:

$$
\bar{D}^{\dot{\alpha}} \Phi^{\dagger} \bar{D}_{\dot{\alpha}} \Phi^{\dagger}=-\bar{D}_{\dot{\alpha}} \Phi^{\dagger} \bar{D}^{\dot{\alpha}} \Phi^{\dagger}=-\left(D^{\alpha} \Phi D_{\alpha} \Phi\right)^{\dagger}
$$

which gives

$$
\bar{D}^{\dot{\alpha}} \Phi^{\dagger} \bar{D}_{\dot{\alpha}} \Phi^{\dagger}=4 F^{* 2} \bar{\theta}^{2}-4 \partial^{\kappa} \phi^{*} \partial_{\kappa} \phi^{*} \theta^{2}+8 i F^{*} \partial_{\kappa} \phi^{*} \theta \sigma^{\kappa} \bar{\theta}-4 \partial^{\mu} F^{*} \partial_{\mu} \phi^{*} \theta^{2} \bar{\theta}^{2}
$$

The product $\left(D^{\alpha} \Phi D_{\alpha} \Phi\right)\left(\bar{D}^{\dot{\alpha}} \Phi^{\dagger} \bar{D}_{\dot{\alpha}} \Phi^{\dagger}\right)$ in terms of component fields is given by

$$
\left(D^{\alpha} \Phi D_{\alpha} \Phi\right)\left(\bar{D}^{\dot{\alpha}} \Phi^{\dagger} \bar{D}_{\dot{\alpha}} \Phi^{\dagger}\right)=16\left(F^{2} F^{* 2}+\partial^{\kappa} \phi \partial_{\kappa} \phi \partial^{\mu} \phi^{*} \partial_{\mu} \phi^{*}-2 F F^{*} \partial^{\mu} \phi \partial_{\mu} \phi^{*}\right) \theta^{2} \bar{\theta}^{2} .
$$

Note that $\left(D^{\alpha} \Phi D_{\alpha} \Phi\right)\left(\bar{D}^{\dot{\alpha}} \Phi^{\dagger} \bar{D}_{\dot{\alpha}} \Phi^{\dagger}\right)$ already contains the highest possible power of the Grassmann numbers, therefore, the factor $G(\Phi)$ multiplying it in the case of the Lagrangian eq. (A.1) can simply be replaced with $G(\phi)$.

Combining terms we get the following expression for the Lagrangian:

$$
\mathcal{L}_{\mathrm{DBI}}=F F^{*}+G(\phi)\left(-2 F F^{*} \partial^{\mu} \phi \partial_{\mu} \phi^{*}+F^{2} F^{* 2}\right)-T A(\phi)+G(\phi) B(\phi) T^{2} .
$$

We can further simplify and write the DBI Lagrangian in the form

$$
\begin{aligned}
\mathcal{L}_{\mathrm{DBI}}= & -T \sqrt{1+2 T^{-1} \partial_{\mu} \phi \partial^{\mu} \phi^{*}+T^{-2}\left(\partial_{\mu} \phi \partial^{\mu} \phi^{*}\right)^{2}-T^{-2}\left(\partial_{\mu} \phi \partial^{\mu} \phi\right)\left(\partial_{\nu} \phi^{*} \partial^{\nu} \phi^{*}\right)} \\
& +T+F F^{*}+G(\phi)\left(-2 F F^{*} \partial^{\mu} \phi \partial_{\mu} \phi^{*}+F^{2} F^{* 2}\right) .
\end{aligned}
$$

\section{B Equations for $F_{k}, F_{k}^{*}(k=1,2)$}

We need to eliminate $F_{k}$ and $F_{k}^{*}$ from the Lagrangian of eq. (3.12) to construct an equation of motion for $\phi_{k}$ only. In order to do that, we first vary with respect to $F_{k}$ and $F_{k}^{*}$ to obtain 
the following equations for $F_{k}$ and $F_{k}^{*}$. Here variations with respect to $F_{1}^{*}$ and $F_{1}$ give

$$
\begin{aligned}
F_{1}+\frac{\partial W^{*}}{\partial \phi_{1}^{*}}+G(\phi)\left[\alpha_{1}\left(-2 F_{1} \partial_{a} \phi_{1} \partial^{a} \phi_{1}^{*}+2 F_{1}^{2} F_{1}^{*}\right)\right. & +\alpha_{2}\left(-2 F_{2} \partial_{a} \phi_{2} \partial^{a} \phi_{1}^{*}+2 F_{2}^{2} F_{1}^{*}\right) \\
& \left.+\alpha_{3}\left(-F_{1} \partial_{a} \phi_{2} \partial^{a} \phi_{2}^{*}+F_{1} F_{2} F_{2}^{*}\right)\right]=0, \\
F_{1}^{*}+\frac{\partial W}{\partial \phi_{1}}+G(\phi)\left[\alpha_{1}\left(-2 F_{1}^{*} \partial_{a} \phi_{1} \partial^{a} \phi_{1}^{*}+2 F_{1} F_{1}^{* 2}\right)\right. & +\alpha_{2}\left(-2 F_{2}^{*} \partial_{a} \phi_{2}^{*} \partial^{a} \phi_{1}+2 F_{2}^{* 2} F_{1}\right) \\
& \left.+\alpha_{3}\left(-F_{1}^{*} \partial_{a} \phi_{2}^{*} \partial^{a} \phi_{2}+F_{1}^{*} F_{2} F_{2}^{*}\right)\right]=0 .
\end{aligned}
$$

Similarly variations with respect to $F_{2}^{*}$ and $F_{2}$ give

$$
\begin{aligned}
F_{2}+\frac{\partial W^{*}}{\partial \phi_{2}^{*}}+G(\phi)\left[\alpha_{1}\left(-2 F_{2} \partial_{a} \phi_{2} \partial^{a} \phi_{2}^{*}+2 F_{2}^{2} F_{2}^{*}\right)\right. & +\alpha_{2}\left(-2 F_{1} \partial_{a} \phi_{1} \partial^{a} \phi_{2}^{*}+2 F_{1}^{2} F_{2}^{*}\right) \\
& \left.+\alpha_{3}\left(-F_{2} \partial_{a} \phi_{1} \partial^{a} \phi_{1}^{*}+F_{2} F_{1} F_{1}^{*}\right)\right]=0, \\
F_{2}^{*}+\frac{\partial W}{\partial \phi_{2}}+G(\phi)\left[\alpha_{1}\left(-2 F_{2}^{*} \partial_{a} \phi_{2} \partial^{a} \phi_{2}^{*}+2 F_{2} F_{2}^{* 2}\right)\right. & +\alpha_{2}\left(-2 F_{1}^{*} \partial_{a} \phi_{1}^{*} \partial^{a} \phi_{2}+2 F_{1}^{* 2} F_{2}\right) \\
& \left.+\alpha_{3}\left(-F_{2}^{*} \partial_{a} \phi_{1}^{*} \partial^{a} \phi_{1}+F_{2}^{*} F_{1} F_{1}^{*}\right)\right]=0 .
\end{aligned}
$$

These give rise to a set of four coupled equations for $F_{1}, F_{1}^{*}, F_{2}, F_{2}^{*}$ which are difficult to solve analytically. To keep the analysis under control we set $\alpha_{2}=0=\alpha_{3}$. In this case the equations for $F_{1}, F_{1}^{*}$ become decoupled from those for $F_{2}, F_{2}^{*}$ and we get

$$
\begin{array}{rlrl}
F_{k}^{*}+\frac{\partial W}{\partial \phi_{k}}+\alpha_{1} G(\phi)\left(-2 F_{k}^{*} \partial^{\mu} \phi_{k} \partial_{\mu} \phi_{k}^{*}+2 F_{k} F_{k}^{* 2}\right) & =0, & k=1,2, \\
F_{k}+\frac{\partial W^{*}}{\partial \phi_{k}^{*}}+\alpha_{1} G(\phi)\left(-2 F_{k} \partial^{\mu} \phi_{k} \partial_{\mu} \phi_{k}^{*}+2 F_{k}^{2} F_{k}^{*}\right)=0, & k=1,2 .
\end{array}
$$

We multiply eq. (B.3) by $F_{k}$ and eq. (B.4) by $F_{k}^{*}$, and subtract one from another from which we can extract an equation for $F_{k}^{*}$ in terms of $F_{k}$ :

$$
F_{k}^{*}=\left(\frac{\partial W}{\partial \phi_{k}} / \frac{\partial W^{*}}{\partial \phi_{k}^{*}}\right) F_{k} .
$$

Substitution back in eq. (B.4) gives an equation for $F_{k}$ :

$$
2 \alpha_{1} G(\phi) \frac{\partial W}{\partial \phi_{k}} F_{k}^{3}+\frac{\partial W^{*}}{\partial \phi_{k}^{*}}\left(1-2 \alpha_{1} G(\phi) \partial^{\mu} \phi_{k} \partial_{\mu} \phi_{k}^{*}\right) F_{k}+\left(\frac{\partial W^{*}}{\partial \phi_{k}^{*}}\right)^{2}=0 .
$$

To simplify this equation we define $p$ and $q$ so that

$$
\begin{aligned}
p_{k} & =\left(\frac{\partial W}{\partial \phi_{k}}\right)^{-1} \frac{\partial W^{*}}{\partial \phi_{k}^{*}} \frac{1-2 \alpha_{1} G \partial_{\mu} \phi_{k} \partial^{\mu} \phi_{k}^{*}}{2 \alpha_{1} G}, \\
q_{k} & =\frac{1}{2 \alpha_{1} G}\left(\frac{\partial W}{\partial \phi_{k}}\right)^{-1}\left(\frac{\partial W^{*}}{\partial \phi_{k}^{*}}\right)^{2} .
\end{aligned}
$$


Substitution of $\partial^{\mu} \phi_{k} \partial_{\mu} \phi_{k}^{*}$ in terms of $p_{k}$ using eq. (B.7) in eq. (B.6) and using eq. (B.8) to eliminate $\left(\frac{\partial W^{*}}{\partial \phi_{k}^{*}}\right)^{2}$ in terms of $q_{k}$ in eq. (B.6) gives

$$
F_{k}^{3}+p_{k} F_{k}+q_{k}=0
$$

where we cancelled a common factor of $2 G \frac{\partial W}{\partial \phi_{k}}$. The equation above is cubic in $F_{k}$ and it might appear that there are three consistent solutions. To see if this is the case we substitute eq. (B.5) back into eq. (3.15) to obtain a solution for $F_{k}^{*}$. Upon simplification, one gets

$$
\begin{aligned}
F_{k}^{*}= & \omega^{j}\left(-\frac{q_{k}^{*}}{2}+\sqrt{\left(\frac{q_{k}^{*}}{2}\right)^{2}+\left(\frac{p_{k}^{*}}{3}\right)^{3}}\right)^{1 / 3} \\
& +\omega^{3-j}\left(-\frac{q_{k}^{*}}{2}-\sqrt{\left(\frac{q_{k}^{*}}{2}\right)^{2}+\left(\frac{p_{k}^{*}}{3}\right)^{3}}\right)^{1 / 3} .
\end{aligned}
$$

Note that this expression is only a complex conjugate of eq. (3.15) if $j=0\left(\omega^{j}\right.$ and $\omega^{3-j}$ will have to be interchanged for it to be a complex conjugate in the case of $j=1$ and $j=2$ ), therefore only $j=0$ corresponds to a solution for the auxiliary fields $F_{k}$.

\section{DBI scalar potential with derivative terms absent}

To discuss the limit of the DBI potential to the standard supersymmetric potential we need to drop the derivative terms on $\phi$ in the potential. In this case the full form of the scalar potential looks very different from the usual supersymmetric potential. To keep the expressions as simple as possible we consider the case of just one scalar field although extension to more fields is straightforward. In this case we have

$$
\begin{aligned}
G & =\frac{1}{2 T}, \\
p & =T\left(\frac{\partial W}{\partial \phi}\right)^{-1} \frac{\partial W^{*}}{\partial \phi^{*}}, \\
q & =T\left(\frac{\partial W}{\partial \phi}\right)^{-1}\left(\frac{\partial W^{*}}{\partial \phi^{*}}\right)^{2},
\end{aligned}
$$

$F$ is given by

$$
\begin{aligned}
F= & T^{1 / 2} \\
& \times\left(\frac{\partial W}{\partial \phi}\right)^{-1 / 3}\left(\frac{\partial W^{*}}{\partial \phi^{*}}\right)^{1 / 2}\left(\left(-\frac{1}{T^{1 / 2}} \sqrt{\frac{1}{4} \frac{\partial W^{*}}{\partial \phi^{*}}}+\sqrt{\frac{1}{27}\left(\frac{\partial W}{\partial \phi}\right)^{-1}+\frac{1}{4 T} \frac{\partial W^{*}}{\partial \phi^{*}}}\right)^{1 / 3}\right. \\
& \left.+\left(-\frac{1}{T^{1 / 2}} \sqrt{\frac{1}{4} \frac{\partial W^{*}}{\partial \phi^{*}}}-\sqrt{\frac{1}{27}\left(\frac{\partial W}{\partial \phi}\right)^{-1}+\frac{1}{4 T} \frac{\partial W^{*}}{\partial \phi^{*}}}\right)^{1 / 3}\right),
\end{aligned}
$$


and the scalar potential is

$$
V(\phi)=-\left(F^{*} F+\frac{\partial W}{\partial \phi} F+\frac{\partial W^{*}}{\partial \phi} F^{*}+\frac{1}{2 T} F^{2} F^{* 2}\right) .
$$

Further, an explicit form of the potential can be gotten by using eq. (C.2) back into the potential eq. (C.3) which gives

$$
V(\phi)=V_{1}(\phi)+V_{2}(\phi)+V_{3}(\phi)+V_{4}(\phi),
$$

where

$$
\begin{aligned}
& V_{1}(\phi)=-T\left(\frac{\partial W}{\partial \phi} \frac{\partial W^{*}}{\partial \phi^{*}}\right)^{1 / 6}\left(Q_{+}+Q_{-}\right)\left(Q_{+}^{*}+Q_{-}^{*}\right), \\
& V_{2}(\phi)=-T^{1 / 2}\left(\frac{\partial W}{\partial \phi}\right)^{2 / 3}\left(\frac{\partial W^{*}}{\partial \phi^{*}}\right)^{1 / 2}\left(Q_{+}+Q_{-}\right), \\
& V_{3}(\phi)=V_{2}^{*}(\phi) \\
& V_{4}(\phi)=-\frac{1}{2} T\left(\frac{\partial W}{\partial \phi} \frac{\partial W^{*}}{\partial \phi^{*}}\right)^{1 / 3}\left(Q_{+}+Q_{-}\right)^{2}\left(Q_{+}^{*}+Q_{-}^{*}\right)^{2},
\end{aligned}
$$

and

$$
Q_{ \pm}=\left(-\frac{1}{T^{1 / 2}} \sqrt{\frac{1}{4} \frac{\partial W^{*}}{\partial \phi^{*}}} \pm \sqrt{\frac{1}{27}\left(\frac{\partial W}{\partial \phi}\right)^{-1}+\frac{1}{4 T} \frac{\partial W^{*}}{\partial \phi^{*}}}\right)^{1 / 3}
$$

We can expand $F$ given by eq. (C.2) in powers of $1 / T$ and this expansion is exhibited in eq. (3.16). Similarly we can expand $V$ given by eq. (C.3) in powers of $1 / T$ and this expansion is given in eq. (3.17). One can see that the lowest terms in the expansion for both $F$ and $V$ give the standard result.

Open Access. This article is distributed under the terms of the Creative Commons Attribution License (CC-BY 4.0), which permits any use, distribution and reproduction in any medium, provided the original author(s) and source are credited.

\section{References}

[1] A.H. Guth, The Inflationary Universe: A Possible Solution to the Horizon and Flatness Problems, Phys. Rev. D 23 (1981) 347 [InSPIRE].

[2] A.A. Starobinsky, A New Type of Isotropic Cosmological Models Without Singularity, Phys. Lett. B 91 (1980) 99 [INSPIRE].

[3] A.D. Linde, A New Inflationary Universe Scenario: A Possible Solution of the Horizon, Flatness, Homogeneity, Isotropy and Primordial Monopole Problems, Phys. Lett. B 108 (1982) 389 [INSPIRE].

[4] A. Albrecht and P.J. Steinhardt, Cosmology for Grand Unified Theories with Radiatively Induced Symmetry Breaking, Phys. Rev. Lett. 48 (1982) 1220 [INSPIRE]. 
[5] K. Sato, First Order Phase Transition of a Vacuum and Expansion of the Universe, Mon. Not. Roy. Astron. Soc. 195 (1981) 467 [INSPIRE].

[6] A.D. Linde, Chaotic Inflation, Phys. Lett. B 129 (1983) 177 [INSPIRE].

[7] V.F. Mukhanov and G.V. Chibisov, Quantum Fluctuations and a Nonsingular Universe, JETP Lett. 33 (1981) 532 [Pisma Zh. Eksp. Teor. Fiz. 33 (1981) 549] [InSPIRE].

[8] S.W. Hawking, The Development of Irregularities in a Single Bubble Inflationary Universe, Phys. Lett. B 115 (1982) 295 [inSPIRE].

[9] A.A. Starobinsky, Dynamics of Phase Transition in the New Inflationary Universe Scenario and Generation of Perturbations, Phys. Lett. B 117 (1982) 175 [INSPIRE].

[10] A.H. Guth and S.Y. Pi, Fluctuations in the New Inflationary Universe, Phys. Rev. Lett. 49 (1982) 1110 [INSPIRE].

[11] J.M. Bardeen, P.J. Steinhardt and M.S. Turner, Spontaneous Creation of Almost Scale-Free Density Perturbations in an Inflationary Universe, Phys. Rev. D 28 (1983) 679 [InSPIRE].

[12] C. Cheung, P. Creminelli, A.L. Fitzpatrick, J. Kaplan and L. Senatore, The Effective Field Theory of Inflation, JHEP 03 (2008) 014 [arXiv:0709.0293] [INSPIRE].

[13] Planck collaboration, Planck 2015 results. I. Overview of products and scientific results, Astron. Astrophys. 594 (2016) A1 [arXiv:1502.01582] [INSPIRE].

[14] Planck collaboration, Planck 2015 results. XX. Constraints on inflation, Astron. Astrophys. 594 (2016) A20 [arXiv: 1502.02114] [INSPIRE].

[15] BICEP2 and Keck ARRAY collaborations, Improved Constraints on Cosmology and Foregrounds from BICEP2 and Keck Array Cosmic Microwave Background Data with Inclusion of 95 GHz Band, Phys. Rev. Lett. 116 (2016) 031302 [arXiv:1510.09217] [INSPIRE].

[16] K. Freese, J.A. Frieman and A.V. Olinto, Natural inflation with pseudo Nambu-Goldstone bosons, Phys. Rev. Lett. 65 (1990) 3233 [INSPIRE].

[17] F.C. Adams, J.R. Bond, K. Freese, J.A. Frieman and A.V. Olinto, Natural inflation: Particle physics models, power law spectra for large scale structure and constraints from COBE, Phys. Rev. D 47 (1993) 426 [hep-ph/9207245] [INSPIRE].

[18] T. Banks, M. Dine, P.J. Fox and E. Gorbatov, On the possibility of large axion decay constants, JCAP 06 (2003) 001 [hep-th/0303252] [INSPIRE].

[19] P. Svrček and E. Witten, Axions In String Theory, JHEP 06 (2006) 051 [hep-th/0605206] [INSPIRE].

[20] J.E. Kim, H.P. Nilles and M. Peloso, Completing natural inflation, JCAP 01 (2005) 005 [hep-ph/0409138] [INSPIRE].

[21] C. Long, L. McAllister and P. McGuirk, Aligned Natural Inflation in String Theory, Phys. Rev. D 90 (2014) 023501 [arXiv: 1404.7852] [INSPIRE].

[22] P. Nath and M. Piskunov, Evidence for Inflation in an Axion Landscape, JHEP 03 (2018) 121 [arXiv: 1712.01357] [INSPIRE].

[23] P. Nath, Supersymmetry, Supergravity, and Unification, Cambridge Monographs On Mathematical Physics, Cambridge University Press, Cambridge U.K. (2016) [INSPIRE]. 
[24] J.M. Maldacena, Non-Gaussian features of primordial fluctuations in single field inflationary models, JHEP 05 (2003) 013 [astro-ph/0210603] [INSPIRE].

[25] D. Seery and J.E. Lidsey, Primordial non-Gaussianities in single field inflation, JCAP 06 (2005) 003 [astro-ph/0503692] [INSPIRE].

[26] D. Seery and J.E. Lidsey, Primordial non-Gaussianities from multiple-field inflation, JCAP 09 (2005) 011 [astro-ph/0506056] [INSPIRE].

[27] X. Chen, Running non-Gaussianities in DBI inflation, Phys. Rev. D 72 (2005) 123518 [astro-ph/0507053] [INSPIRE].

[28] X. Chen, M.-x. Huang, S. Kachru and G. Shiu, Observational signatures and non-Gaussianities of general single field inflation, JCAP 01 (2007) 002 [hep-th/0605045] [INSPIRE].

[29] D.H. Lyth and Y. Rodriguez, The Inflationary prediction for primordial non-Gaussianity, Phys. Rev. Lett. 95 (2005) 121302 [astro-ph/0504045] [INSPIRE].

[30] M. Alishahiha, E. Silverstein and D. Tong, DBI in the sky, Phys. Rev. D 70 (2004) 123505 [hep-th/0404084] [INSPIRE].

[31] D.A. Easson, R. Gregory, D.F. Mota, G. Tasinato and I. Zavala, Spinflation, JCAP 02 (2008) 010 [arXiv:0709.2666] [INSPIRE].

[32] M.-x. Huang, G. Shiu and B. Underwood, Multifield DBI Inflation and Non-Gaussianities, Phys. Rev. D 77 (2008) 023511 [arXiv:0709.3299] [INSPIRE].

[33] C. Gordon, D. Wands, B.A. Bassett and R. Maartens, Adiabatic and entropy perturbations from inflation, Phys. Rev. D 63 (2001) 023506 [astro-ph/0009131] [INSPIRE].

[34] D. Langlois, S. Renaux-Petel, D.A. Steer and T. Tanaka, Primordial fluctuations and non-Gaussianities in multi-field DBI inflation, Phys. Rev. Lett. 101 (2008) 061301 [arXiv:0804.3139] [INSPIRE].

[35] N. Arkani-Hamed, H.-C. Cheng, P. Creminelli and L. Randall, Pseudonatural inflation, JCAP 07 (2003) 003 [hep-th/0302034] [INSPIRE].

[36] D.E. Kaplan and N.J. Weiner, Little inflatons and gauge inflation, JCAP 02 (2004) 005 [hep-ph/0302014] [INSPIRE].

[37] D. Green, B. Horn, L. Senatore and E. Silverstein, Trapped Inflation, Phys. Rev. D 80 (2009) 063533 [arXiv:0902.1006] [inSPIRE].

[38] T. Higaki and F. Takahashi, Natural and Multi-Natural Inflation in Axion Landscape, JHEP 07 (2014) 074 [arXiv: 1404.6923] [INSPIRE].

[39] T. Higaki and F. Takahashi, Axion Landscape and Natural Inflation, Phys. Lett. B 744 (2015) 153 [arXiv: 1409.8409] [INSPIRE].

[40] K. Kadota, T. Kobayashi, A. Oikawa, N. Omoto, H. Otsuka and T.H. Tatsuishi, Small field axion inflation with sub-Planckian decay constant, JCAP 10 (2016) 013 [arXiv:1606.03219] [INSPIRE].

[41] T. Kobayashi, A. Oikawa, N. Omoto, H. Otsuka and I. Saga, Constraints on small-field axion inflation, Phys. Rev. D 95 (2017) 063514 [arXiv:1609.05624] [INSPIRE].

[42] S. Kachru, R. Kallosh, A.D. Linde, J.M. Maldacena, L.P. McAllister and S.P. Trivedi, Towards inflation in string theory, JCAP 10 (2003) 013 [hep-th/0308055] [INSPIRE]. 
[43] J.J. Blanco-Pillado et al., Racetrack inflation, JHEP 11 (2004) 063 [hep-th/0406230] [INSPIRE].

[44] M. Cicoli, K. Dutta, A. Maharana and F. Quevedo, Moduli Vacuum Misalignment and Precise Predictions in String Inflation, JCAP 08 (2016) 006 [arXiv:1604.08512] [InSPIRE].

[45] E. Pajer and M. Peloso, A review of Axion Inflation in the era of Planck, Class. Quant. Grav. 30 (2013) 214002 [arXiv: 1305.3557] [INSPIRE].

[46] D.J.E. Marsh, Axion Cosmology, Phys. Rept. 643 (2016) 1 [arXiv:1510.07633] [InSPIRE].

[47] A. Ernst, A. Ringwald and C. Tamarit, Axion Predictions in $\mathrm{SO}(10) \times \mathrm{U}(1)_{\mathrm{PQ}}$ Models, JHEP 02 (2018) 103 [arXiv: 1801.04906] [INSPIRE].

[48] S. Dimopoulos, S. Kachru, J. McGreevy and J.G. Wacker, N-flation, JCAP 08 (2008) 003 [hep-th/0507205] [INSPIRE];

[49] A.R. Liddle, A. Mazumdar and F.E. Schunck, Assisted inflation, Phys. Rev. D 58 (1998) 061301 [astro-ph/9804177] [INSPIRE].

[50] J. Khoury, J.-L. Lehners and B. Ovrut, Supersymmetric $P(X, \phi)$ and the Ghost Condensate, Phys. Rev. D 83 (2011) 125031 [arXiv: 1012.3748] [INSPIRE].

[51] J. Khoury, J.-L. Lehners and B.A. Ovrut, Supersymmetric Galileons, Phys. Rev. D 84 (2011) 043521 [arXiv: 1103.0003] [INSPIRE].

[52] D. Baumann and D. Green, Signatures of Supersymmetry from the Early Universe, Phys. Rev. D 85 (2012) 103520 [arXiv:1109.0292] [INSPIRE].

[53] D. Baumann and D. Green, Supergravity for Effective Theories, JHEP 03 (2012) 001 [arXiv: 1109.0293] [INSPIRE].

[54] M. Roček and A.A. Tseytlin, Partial breaking of global D $=4$ supersymmetry, constrained superfields and three-brane actions, Phys. Rev. D 59 (1999) 106001 [hep-th/9811232] [INSPIRE].

[55] A.A. Tseytlin, Born-Infeld action, supersymmetry and string theory, in The many faces of the superworld, M.A. Shifman ed., World Scientific (2000), pp. 417-452 [hep-th/9908105] [INSPIRE].

[56] K. Ito, H. Nakajima and S. Sasaki, Deformation of super Yang-Mills theories in RR 3-form background, JHEP 07 (2007) 068 [arXiv:0705.3532] [INSPIRE].

[57] M. Billó, L. Ferro, M. Frau, F. Fucito, A. Lerda and J.F. Morales, Flux interactions on D-branes and instantons, JHEP 10 (2008) 112 [arXiv:0807.1666] [INSPIRE].

[58] S. Sasaki, M. Yamaguchi and D. Yokoyama, Supersymmetric DBI inflation, Phys. Lett. B 718 (2012) 1 [arXiv:1205.1353] [INSPIRE].

[59] S. Aoki and Y. Yamada, More on DBI action in $4 D \mathcal{N}=1$ supergravity, JHEP 01 (2017) 121 [arXiv: 1611.08426] [INSPIRE].

[60] J. Halverson, C. Long and P. Nath, Ultralight axion in supersymmetry and strings and cosmology at small scales, Phys. Rev. D 96 (2017) 056025 [arXiv:1703.07779] [InSPIRE].

[61] J. Garriga and V.F. Mukhanov, Perturbations in k-inflation, Phys. Lett. B 458 (1999) 219 [hep-th/9904176] [INSPIRE].

[62] C. Armendariz-Picon, T. Damour and V.F. Mukhanov, k-inflation, Phys. Lett. B 458 (1999) 209 [hep-th/9904075] [INSPIRE]. 
[63] V. Acquaviva, N. Bartolo, S. Matarrese and A. Riotto, Second order cosmological perturbations from inflation, Nucl. Phys. B 667 (2003) 119 [astro-ph/0209156] [INSPIRE].

[64] P. Creminelli, On non-Gaussianities in single-field inflation, JCAP 10 (2003) 003 [astro-ph/0306122] [INSPIRE].

[65] E. Silverstein and D. Tong, Scalar speed limits and cosmology: Acceleration from D-cceleration, Phys. Rev. D 70 (2004) 103505 [hep-th/0310221] [INSPIRE].

[66] A. Gruzinov, Consistency relation for single scalar inflation, Phys. Rev. D 71 (2005) 027301 [astro-ph/0406129] [INSPIRE].

[67] P. Creminelli, A. Nicolis, L. Senatore, M. Tegmark and M. Zaldarriaga, Limits on non-Gaussianities from WMAP data, JCAP 05 (2006) 004 [astro-ph/0509029] [INSPIRE].

[68] D. Babich, P. Creminelli and M. Zaldarriaga, The Shape of non-Gaussianities, JCAP 08 (2004) 009 [astro-ph/0405356] [INSPIRE].

[69] PlancK collaboration, Planck 2015 results. XVII. Constraints on primordial non-Gaussianity, Astron. Astrophys. 594 (2016) A17 [arXiv:1502.01592] [INSPIRE].

[70] E. Komatsu and D.N. Spergel, Acoustic signatures in the primary microwave background bispectrum, Phys. Rev. D 63 (2001) 063002 [astro-ph/0005036] [INSPIRE].

[71] WMAP collaboration, First year Wilkinson Microwave Anisotropy Probe (WMAP) observations: tests of Gaussianity, Astrophys. J. Suppl. 148 (2003) 119 [astro-ph/0302223] [INSPIRE].

[72] L. Verde, L.-M. Wang, A. Heavens and M. Kamionkowski, Large scale structure, the cosmic microwave background and primordial non-Gaussianity, Mon. Not. Roy. Astron. Soc. 313 (2000) L141 [astro-ph/9906301] [INSPIRE].

[73] S. Kachru, R. Kallosh, A.D. Linde and S.P. Trivedi, de Sitter vacua in string theory, Phys. Rev. D 68 (2003) 046005 [hep-th/0301240] [INSPIRE].

[74] V. Balasubramanian, P. Berglund, J.P. Conlon and F. Quevedo, Systematics of moduli stabilisation in Calabi-Yau flux compactifications, JHEP 03 (2005) 007 [hep-th/0502058] [INSPIRE]. 